\title{
Grammatikvermittlung in neueren kroatischen DaF-Lehrwerken
}

\author{
Maja Häusler/Zrinjka Glovacki-Bernardi (Zagreb)
}

\begin{abstract}
The first part of the article deals with the presentation of grammar from the linguistic point of view. It gives the results of the analysis of the presentation of grammar in textbooks for German as a foreign language written by Croatian authors (in textbooks for elementary school, grammar school; for German as the first and the second foreign language). The analysis is carried out under the following criteria: explicit or implicit presentation of grammar, theoretical grammatical foundations, usage of metalinguistic terms, the language used for explanations. The second part of the article presents the analysis of grammar teaching in selected textbooks under didactical and methodical aspects (usage in texts, types of exercises and assignments). The results of the analysis are evaluated in terms of the supposed appropriateness and contemporary views regarding the grammar teaching in teaching foreign languages. What we are also interested in is the question whether and to what extent the requirements of the common international competencies description for the exam certificate have modified or changed the significance of grammar teaching in Croatian textbooks for German as a foreign language.
\end{abstract}

\section{$1 \quad$ Einleitung}

Trotz unterschiedlicher Ansätze und Unterrichtsmethoden wird im Fremdsprachenunterricht immer auch Grammatik vermittelt - Formen und Strukturen, Relationen zwischen den sprachlichen Zeichen, Kombinationsmöglichkeiten. Diese Art von Grammatik ist keine vereinfachte Version eines ausgewählten Grammatik- beziehungsweise Sprachmodells, sondern immer nur ein Mittel zur optimalen Realisierung bestimmter Lernziele.

Obwohl in theoretischen Auseinandersetzungen, besonders seitens der Spracherwerbsforschung, der Nutzen der Grammatikunterweisung immer wieder in Frage gestellt wird, ${ }^{1}$ ist Fremdsprachenunterricht ohne Grammatikvermittlung nicht vorstellbar. Zum einen identifiziert das allgemeine Publikum, und somit auch die Zielgruppe der Lerner, immer noch das Sprachenlernen mit dem Erlernen der Grammatik einer Sprache. Zum anderen bildet die Grammatik das Gerüst, an dem entlang die Autoren ihre Lehrwerke konzipieren, da Versuche, die Lehrwerke nach anderen Kategorien zu entwickeln, zu keinen brauchbaren Ergebnissen führten.

Entsprechend den jeweiligen übergreifenden Zielsetzungen räumen die Methoden des Fremdsprachenunterrichts der Grammatikvermittlung unterschiedlichen Stellenwert ein. Eine wichtige Rolle bei den Veränderungen der Lernziele spielt die Anwendung linguistischer Erkenntnisse: Mit der Ausweitung des Interesses linguistischer Spracherforschung bekommt auch die Grammatik in den Lehrwerken neue Akzente und andere Inhalte.

\footnotetext{
${ }^{1}$ Zur Diskussion von theoretischen Positionen bezüglich der Frage, wie man die Grammatik einer Fremdsprache erwirbt, vgl. etwa Diehl 2000: 44-49.
} 
Für diesen Beitrag haben wir die Grammatikvermittlung in neueren Lehrwerken für Deutsch als Fremdsprache, die in Kroatien erschienen sind, der linguistischen und der fachdidaktischen Analyse unterzogen. ${ }^{2}$ Da es sich um Lehrwerke handelt, die für den Gebrauch in kroatischen Schulen verfasst und auch zugelassen wurden, geben wir einleitend einige Erläuterungen zum Deutschen als Fremdsprache im kroatischen Schulsystem.

\section{Deutsch als Fremdsprache im kroatischen Schulsystem}

Die deutsche Sprache kann an kroatischen Schulen als erste, zweite oder dritte Fremdsprache gelernt werden. Die Grundschule umfasst in Kroatien acht Klassenstufen, daran schließen vierjährige Gymnasien und drei- oder vierjährige Mittelschulen mit Fachausbildung an.

Mit Deutsch als erster Fremdsprache fangen die Schüler in der ersten Klassenstufe an und lernen es bis zum Abitur. In den Klassen 1 bis 4 haben sie 2 Wochenstunden, danach 3 . Auch in Gymnasien lernen die Schüler ihre erste Fremdsprache mit 3 Stunden wöchentlich, in Sprachgymnasien mit 4. In den meisten Mittelschulen mit Fachausbildung wird die erste Fremdsprache nur mit 2 Wochenstunden gelernt.

Mit Deutsch als zweiter Fremdsprache fangen die Schüler in der 4. Grundschulklasse an, in allen Klassen (4 bis 8) haben sie 2 Stunden wöchentlich. Deutsch als zweite Fremdsprache können die Schüler auch in der Mittelschule zu lernen anfangen. Sie haben in der Regel 2 Wochenstunden in allen vier Klassen. An Sprachgymnasien haben die Lerner des Deutschen als zweiter Fremdsprache in der ersten Klasse 4 Stunden, danach 3. Auch an einigen Fachschulen, mit Ausrichtung für Berufe in der Wirtschaft und Verwaltung, im Tourismus oder Hotelwesen, stehen mehr Stunden für den fremdsprachlichen Unterricht sowohl der ersten als auch der zweiten Fremdsprache zur Verfügung. ${ }^{3}$

Lehrwerke für Schulen des kroatischen Bildungssystems werden vom Ministerium nach Gutachtung durch Fachleute für den Gebrauch in bestimmten Schulen zugelassen. Neben den Lehrwerken, die von kroatischen Autorinnen und Autoren verfasst wurden, finden sich unter den für den Deutschunterricht in Kroatien zugelassenen Lehrwerken auch Publikationen deutscher Verlage, die für kroatische Schulen adaptiert wurden.

Die Deutschlehrer einzelner Schulen wählen unter den zugelassenen Lehrwerken jene aus, die sie mit ihren Lernergruppen einsetzen wollen. Das Ministerium sammelt die Daten und stellt Lehrwerke für Grundschulen zur Verfügung, in Zukunft auch für die Mittelschulen. Das Ministerium veröffentlicht die Daten über zugelassene Lehrwerke und auch die Angaben dazu, welche Lehrwerke von welchen Schulen gewählt wurden und dort eingesetzt werden.

Der Marktführer für DaF-Lehrwerke ist immer noch der älteste und angesehenste kroatische Verlag für pädagogische Literatur, Školska knjiga (= Schulbuchverlag), der für den Deutschunterricht ausschließlich einheimische Lehrwerke publiziert. Um die Gunst von Lehrerinnen und Lehrern bemühen sich seit einigen Jahren weitere, neu gegründete Verlage, die auch ihr

\footnotetext{
2 Die Aspekte, nach denen die linguistische und die fachdidaktische Analyse der Lehrwerke vorgenommen wurden, werden am Anfang der entsprechenden Teile des Beitrags angegeben (Punkt 3 und 4). Erarbeitet wurden diese Aspekte aufgrund der einschlägigen Fachliteratur zur Lehrwerkanalyse und zum Grammatikunterricht, wie z. B. des Mannheimer Gutachtens (vgl. Engel et al. 1978). Einige weitere relevante Werke werden im Literaturverzeichnis angeführt (Krumm 1982, Zimmermann 1990, Funk/Koenig 1991, Kast/Neuner 1994, Storch 1999). Als Parameter dienten uns die heute vorherrschenden Erwartungen an Grammatikpräsentation und vermittlung, wie sie aus den Prinzipien der zeitgenössischen Fremdsprachendidaktik abgeleitet werden. Sie werden einleitend zu jedem Punkt der Analyse erläutert.

${ }^{3}$ In den Schulen Kroatiens lernt ein Drittel der Schüler Deutsch als erste oder zweite Fremdsprache (33\%), in den Grundschulen sind es 28.72\%, in den Mittelschulen (einschließlich Gymnasien) 40.95\%. Deutsch als erste Fremdsprache lernten im Schuljahr 2006/07 in der 1. Klasse der Grundschule 11.66\% der Schüler (Englisch: $87.42 \%$, Italienisch und Französisch: unter einem Prozent).
} 
Programm für Deutsch als Fremdsprache ausbauen und gern ins Geschäft mit adaptierten ausländischen Lehrwerken einsteigen.

\section{$3 \quad$ Linguistische Analyse}

In den Lehrwerken, die derzeit in den Schulen Kroatiens eingesetzt werden, wurden aus linguistischer Sicht folgende Aspekte untersucht: die den Lehrwerken zugrunde liegenden Grammatiktheorien; die berücksichtigten linguistischen Ebenen; die eingeführte Terminologie (deutsche/kroatische Bezeichnungen); die explizite/implizite Darstellung grammatischer Inhalte.

\subsection{Lehrwerke für Grundschulen}

Die Lehrwerkserie Deutsch? Super! (Bernardi-Britvec/Kruhan/Salopek 1996; Bernardi-Britvec/Salopek 1998, 1999; Salopek/Bernardi-Britvec 1998; Salopek/Kruhan/Bernardi-Britvec 1997) wurde für Lerner verfasst, die mit Deutsch als erster Fremdsprache in der vierten Klasse der Grundschule anfangen. Im ersten Buch, für das erste Lernjahr, werden zuerst die morphologischen Kategorien schematisch dargestellt, die das Kroatische als grammatische Kategorien nicht kennt - der bestimmte und der unbestimmte Artikel:

$\begin{array}{lll}\text { der Bleistift } & \text { - ein Bleistift } & \text { DER/EIN } \\ \text { die Schultasche } & \text { - eine Schultasche } & \text { DIE/EINE } \\ \text { das Heft } & - \text { ein Heft } & \text { DAS/EIN }\end{array}$

(Bernardi-Britvec/Kruhan/Salopek 1996: 11)

Danach folgen Verbalparadigmen, Präsens von heißen und spielen; gesondert werden paradigmatisch nur die Endungen dargestellt.

Im zweiten Teil des Lehrwerks wird dann die syntaktische Ebene eingeführt und Verbalparadigmen werden durch Beispielsätze präsentiert:

Ich fahre nach Berlin.

Du fährst nach Deutschland.

Mein Vater fährt nach Österreich.

Meine Mutter fährt nach Wien.

Das Kind fährt zu Oma und Opa.

Wir fahren nach Dubrovnik.

Kinder, fahrt ihr auch?

Die Kinder fahren ans Meer. (ebd.: 41)

$\mathrm{Zu}$ berücksichtigen ist, dass das Grundschema dem traditionellen Verbalparadigma entspricht, in dem in der 3. P. Sing. Präs. drei Pronomina - er, sie, es - angeführt werden, daher auch drei Beispielsätze für die 3. P. Sing. Präs., obwohl wiederholt ein und dieselbe Verbalform angeführt wird. Beide Darstellungsmöglichkeiten finden wir auch in den Lehrbüchern der Serie Deutsch? Super! für das zweite und dritte Lernjahr, d. h. für die 5. und 6. Grundschulklasse. In Paradigmen von schlafen, sehen und helfen wird der Vokalwechsel graphisch hervorgehoben, aber keine Erklärungen werden gegeben.

Grammatische Termini sind die Termini der traditionellen Grammatik auf Deutsch, beispielsweise: Das Perfekt von regelmäßigen Verben, Imperativ, Höflichkeitsform, die Deklination des Adjektivs nach dem bestimmten Artikel. Auch die Bildung von Partizip II wird schematisch durch graphische Mittel dargestellt, z. B. ge + lernen $+\mathrm{t}$ (Salopek/Bernardi-Britvec 1998: 40).

Grammatische Erläuterungen auf Deutsch findet man im Lehrwerk für die 8. Grundschulklasse: "Das Futur bezeichnet ein Geschehen in der Zukunft. Häufig steht für die Zukunft das 
Präsens mit Zeitangaben (morgen, nächste Woche)." (Bernardi-Britvec/Salopek 1999: 31) In diesem Lehrwerk findet man auch eine Tabelle mit starken (unregelmäßigen) Verben.

Nachdem für die Grundschule neue Curricula eingeführt wurden, die den Fremdsprachenbeginn in der 1. Klasse der Grundschule verbindlich vorschreiben und die zu erreichenden Kompetenzen nach dem Vorbild der Referenzstufen des Gemeinsamen Europäischen Referenzrahmens für Sprachen formulieren, haben die Autorinnen Bernardi Britvec und Salopek die Lehrwerkserie Deutsch? Super! den Grundprinzipien des neuen Lehrplans angepasst und für die gleiche Zielgruppe (10- bis 11-jährige Lerner, die mit Deutsch nun als zweiter Fremdsprache in der 4. Klasse anfangen) eine neue Version unter dem Titel Flink mit Deutsch (Bernardi-Britvec/Salopek 2007a, 2007b, 2007c; Salopek/Bernardi-Britvec 2007a, 2007b) vorgelegt. Das Modell der Verbalpardigmen nach Mustern der traditionellen Grammatik wird beibehalten. Neu ist die Berücksichtigung der phonetischen Ebene auf kontrastiver Grundlage.

$\mathrm{Ab}$ dem zweiten Lernjahr (5. Grundschulklasse) werden auch Übungen eingeführt, in denen eine Analyse vorausgesetzt wird, die zur Bildung von Regeln führen soll. Es handelt sich um eine Kombination von traditionellem Ansatz (Regeln bilden) und einer Art kognitivem Ansatz, da die Lernenden aufgrund einer Analyse von Beispielen zu bestimmten Schlussfolgerungen kommen sollen. Die Aufgabe lautet:

Ordne und systematisiere. Finde die Regel.

1. Ich ... nicht gut hören. Ich kann das Buch lesen.

2. Du ... Du kannst schnell schreiben.

(Salopek/Bernardi-Britvec 2007a: 38) ${ }^{4}$

Man findet aber auch rein schematische Darstellungen:

Possessivpronomen

$\begin{array}{llll} & \text { ihr Sohn } & & \text { sein Sohn } \\ \text { sie } & \text { ihre Tochter } & \text { er } & \text { seine Tochter } \\ & \text { ihr Kind } & & \text { sein Kind (ebd.: 31) }\end{array}$

Schematische Darstellungen und kognitiver Ansatz werden häufig miteinander kombiniert, so dass beispielsweise einem Deklinationsparadigma die Aufgabe folgt:

S O S

Sammle alle Substantive. Ordne sie. Systematisiere sie nach dem Kasus in dein Heft. (Salopek/

Bernardi-Britvec 2007b: 14)

Im Anhang findet man Paradigmen in Übersicht, beispielsweise Tabellen mit starken Verba. Für die syntaktische Ebene findet man explizite Erläuterungen bzw. Regeln:

Im Nebensatz steht das Verb am Ende des Nebensatzes. (Bernardi-Britvec/Salopek 2007b: 77)

Deutsch lernen - Deutsch spielen (Vajda/Nigl 2006, 2007; Velički/Matolek Veselić 2007a, 2007b; Velički/Vitez 2007). Diese Lehrwerkserie bringt zahlreiche Paradigmen. Hier finden wir im Lehrbuch für das erste Lernjahr im Verbalparadigma als 3. Person Plural auch die Höflichkeitsform: sie singen, Sie singen. Später verschwindet dann diese Form. Einige Aufgaben und schematische Darstellungen sind schwer verständlich, z. B.:

\footnotetext{
${ }^{4}$ Hier und in den folgenden Beispielen bedeuten drei Punkte, dass die Schüler selber weiterdenken, sich eine Regel selber erschließen oder etwas mündlich ergänzen sollen. Linien bedeuten eine Auslassung im Arbeitsheft, wo die Schüler Übungen schriftlich ergänzen sollen.
} 
Perfekt Präsens von haben + Partizip Perfekt

Ich habe gekocht

(Velički/Vitez 2007: 37)

In diesem Beispiel fehlen Anweisungen zur Bildung von Partizip Perfekt. Im Deklinationsparadigma des Personalpronomens werden Nominativ, Dativ und Akkusativ angeführt, obwohl alle anderen Deklinationsparadigmen aus vier Kasus bestehen. Bei der Wiederholung von Perfektbildung unterscheidet man drei Gruppen von Verba: regelmäßige, unregelmäßige und Verba mit sein, obwohl die Kriterien für die ersten zwei Gruppen anders sind als für die dritte Gruppe.

Hurra! Deutsch! (Salopek/Tomljenović-Biškupić 2007a, 2007b; Tomljenović-Biškupić/Salopek 2007a, 2007b, 2007c) ist eine Lehrbuchserie für Deutsch als erste Fremdsprache und zwar ab dem ersten Schuljahr. Da diese Lehrbuchserie für den Früherwerb bestimmt ist, finden wir in den ersten drei Büchern (erste bis dritte Klasse Grundschule) ausschließlich implizite Angaben zur Grammatik. Im ersten Jahr betreffen diese vorwiegend die Graphemik (z. B.: "Das ist ein Fro _ [sch]" (Tomljenović-Biškupić/Salopek 2007a, AH5: 8)), die Morphologie (z. B. Singular und Plural) und die Bildung einfacher Sätze (z. B.: "Das Heft ist grün." (ebd.: 18) "Hast du eine Schwester?" (ebd.: 25)).

Im dritten Lernjahr wird auch die pragmatische Ebene eingeführt, wiederum implizit:

Führt Gespräche

Entschuldige, bitte.

Wo ist der Zoo?

Es tut mit leid, ich weiß es nicht.

(Salopek/Tomljenović-Biškupić 2007a: 15)

Auf dieser Ebene wird der Begriff des Satzes eingeführt, wobei implizit auch die Satzgliedfolge und die grammatische Kongruenz dargestellt werden:

Schreib die Sätze in dein Heft

$\begin{array}{llll}\text { Die Oma } & \text { geht } & \text { die Treppe } & \text { hinauf. } \\ \text { Die Kinder } & \text { gehen } & \text { den Berg } & \text { hinunter. } \\ \text { Er } & \text { läuft. } & & \end{array}$

(ebd., AH: 17)

Im Übungsbuch für das vierte Lernjahr finden wir dann explizite syntaktische Aufgaben, z. B.: "Ordne diese Wörter ein und bilde Sätze." (Tomljenović-Biškupić/Salopek 2007c, AH: 9)

In den ersten vier Lernjahren werden alle Aufgaben sowohl auf Deutsch als auch auf Kroatisch angeführt; ab dem fünften Lernjahr nur auf Deutsch.

\subsection{Lehrwerke für Gymnasien und übrige Mittelschulen}

Die Lehrwerkserie Kontaktsprache Deutsch neu (Häusler 2004; Häusler/Bračun/Kovačić 2001; Häusler/Kern-Francetić 1998, 2000) wendet sich an Lerner, die Deutsch als erste Fremdsprache seit der Grundschule lernen und im Gymnasium ihr 6. bis 9. Lernjahr absolvieren. Grammatische Inhalte werden gesondert in Grammatiktafeln präsentiert. Dargestellt werden vor allem Einheiten der morphologischen und der syntaktischen Ebene. Erläuterungen sind auf Deutsch, z. B.: "An zweiter Stelle steht die konjugierte Verbform." (Häusler/KernFrancetić 1998: 18)

In Kontaktsprache Deutsch 2 neu (siebtes Lernjahr) wird die pragmatische Ebene eingeführt:

\footnotetext{
${ }^{5} \mathrm{AH}=$ Arbeitsheft.
} 
Hörersignale

Während des Gesprächs zeigt der Hörer gewöhnlich an, dass er zuhört. Etwa so:

Ja.

Mhm.

So?

Wirklich wahr? (Häusler/Kern-Francetić 2000: 19)

Die grammatischen Schwerpunkte werden auch deskriptiv erläutert:

Umgangssprache

In der Umgangssprache, besonders der gesprochenen, fangen die Sätze häufig mit dem Verb an: Ist nicht so schlimm. (= Es ist nicht so schlimm.)

Stimmt ja gar nicht. (= Das stimmt ja gar nicht.)

Das finite Verb in der 1. Person Präsens hat manchmal keine Endung:

Find ich auch. (= Finde ich auch.)

Mach ich. (= Ich mache das.)

Es und das Hilfsverb werden ausgelassen:

Nix passiert. (= Es ist nichts passiert.)

(Häusler/Kern-Francetić 2000: 51)

In Kontaktsprache Deutsch 3 neu (achtes Lernjahr) werden in einer der Grammatiktafeln Valenzrelationen dargestellt:

Die meisten Verben verlangen im Satz von ihnen abhängige Substantive oder Pronomen in einem bestimmten Fall (Akkusativ, Dativ, Genitiv oder Präpositionalkasus). (Häusler/Bračun/Kovačić 2001: 82)

Die Darstellung ist auch durch Sprachvergleich charakterisiert:

Die Verben, die den Dativ regieren, haben häufig in der kroatischen Sprache dieselbe Rektion: gefallen, gelingen, gratulieren, helfen, vertrauen, zuhören u. a. (Häusler/Bračun/Kovačić 2001: 82)

In Kontaktsprache Deutsch 4 neu (neuntes Lernjahr) wird die Textebene eingeführt. Unterschiedliche Textsorten werden analysiert und produziert (Anzeigen, Werbetexte, Vorschriften).

In der Lehrwerkserie für Deutsch als zweite Fremdsprache Deutsch für heute und morgen (Marčetić 1997, 1998, 1999, 2000; für Deutsch als zweite Fremdsprache) werden grammatische Inhalte in den Arbeitsbüchern der Serie dargestellt und erläutert. Die Sprache der Erläuterungen ist Kroatisch. Die Erläuterungen sind in der Regel umfangreich:

Izrična rečenica koja sadrži neupravni govor upotrebljava se kao zavisna rečenica s veznikom dass ili kao zavisna rečenica koja nije uvedena veznikom pa joj je red riječi kao u jednostavnoj rečenici. ${ }^{6}$ (Marčetić 2000, AH: 41)

Kada na njemačkom jeziku želimo reći: jedan, jedna, jedno od, izražavamo to za muški i srednji rod produljenim oblikom neodređenoga člana: einer, eines; samo u ženskome rodu neodređeni je član nepromijenjen: eine. (ebd.: 15)

Isto pravilo vrijedi i za negaciju kein: ispred genitiva plurala stoje oblici keiner, keines za muški i srednji rod te keine za ženski rod.7 (ebd.)

\footnotetext{
6 Übersetzung: Der Aussagesatz, in dem indirekte Rede vorkommt, wird als ein Nebensatz mit der Konjunktion dass verwendet oder wie ein Nebensatz, der mit keiner Konjunktion anfängt, so dass die Wortfolge der Wortfolge in einem einfachen Satz entspricht. (Die kroatischen Belege aus den Lehrwerken wurden von den Autorinnen des vorliegenden Beitrags übersetzt und in Fußnoten oder in eckigen Klammern angeführt.)
} 
Deutsch international (Weigmann/Bieler/Schenk 2000a, 2000b, 2000c, 2000d) ist ebenfalls ein Lehrwerk für Deutsch als zweite Fremdsprache in Mittelschulen. Hier werden die traditionelle grammatische Terminologie und paradigmatische Darstellungen verwendet. Explizite grammatische Erläuterungen erfolgen auf Kroatisch:

Već ste naučili glagole kommen i heißen. Oni su u prezentu pravilni uz malu iznimku kod glagola heißen. ${ }^{8}$ (Weigmann/Bieler/Schenk 2000a: 14)

und werden mit einer Analyse kombiniert:

Potražite te oblike u tekstovima A i B. Sastavite tablicu za kommen i heißen u jednini.

U kojem licu glagol heißen nije potpuno pravilan? ${ }^{9}$ (ebd.)

Auf der phonetischen Ebene werden Wort- und Satzakzent eingeübt (vgl. Weigmann/Bieler/Schenk 2000a: 19, 21).

Der analytische Ansatz gilt auch für die syntaktische Ebene:

Usporedite rečenice. Što je različito? [= Vergleicht die Sätze. Was ist unterschiedlich?]

Stefan lernt Mathematik.

Er hat eine Prüfung.

Stefan lernt Mathematik, weil er eine Prüfung hat. (Weigmann/Bieler/Schenk 2000a:

72)

Im Anhang findet man neben einer Liste der unregelmäßigen Verba auch Hinweise auf der pragmatischen Ebene, die mit der Überschrift "Deutsch im Unterricht" in den Kontext des Deutschunterrichts placiert sind:

Um Wiederholung bitten

Wie bitte?

Können Sie/Kannst du das noch mal sagen/erklären?

Etwas langsamer, bitte!/Nicht so schnell! (Weigmann/Bieler/Schenk 2000a: 72)

Im Lehrbuch Deutsch international für das zweite Lernjahr kann man auch den folgenden Hinweis finden:

Riječ particip potječe od latinskoga glagola koji znači 'sudjelovati'. Particip je glagolski oblik koji zajedno s pomoćnim glagolom sudjeluje u tvorbi perfekta. ${ }^{10}$ (Weigmann/Bieler/Schenk 2000b: 33)

Dies ist das einzige Beispiel für eine etymologische Erklärung in allen analysierten Lehrwerken. Der Anhang aus dem ersten Lehrbuch wird wiederholt.

Im dritten Lernjahr findet man auch präskriptive Hinweise:

U govornom se jeziku umjesto genitiva češce upotrebljava dativ s prijedlogom von: ${ }^{11}$ Die Kritik von den Umweltschützern finde ich blöd. (Weigmann/Bieler/Schenk 2000c: 16)

Für Wortbildungsmuster werden Beispiele ohne Erläuterung angeführt:

\footnotetext{
7 Übersetzung: Wenn wir auf Deutsch einer, eine, eines von sagen wollen, drücken wir das für das männliche und sächliche Geschlecht durch eine verlängerte Form des unbestimmten Artikels aus: einer, eines; nur im weiblichen Geschlecht ist der unbestimmte Artikel unverändert: eine.

Dieselbe Regel gilt auch für die Negation kein: vor dem Genitiv Plural stehen die Formen keiner, keines für das männliche und das sächliche Geschlecht und keine für das weibliche Geschlecht.

8 Übersetzung: Ihr habt schon die Verba kommen und heißen gelernt. Im Präsens sind sie regelmäßig mit einer kleinen Ausnahme beim Verb heißen.

9 Übersetzung: Findet diese Formen in den Texten A und B. Bildet eine Tabelle für kommen und heißen im Singular. In welcher Person ist das Verb heißen nicht ganz regelmäßig?

10 Übersetzung: Das Wort Partizip stammt von einem lateinischen Verb, das 'teilnehmen' bedeutet. Das Partizip ist eine Verbform, die zusammen mit einem Hilfsverb an der Bildung von Perfekt teilnimmt.

11 Übersetzung: In der Umgangssprache wird anstatt Genitiv häufig Dativ mit der Präposition von verwendet.
} 
Liebeslied sich verlieben lieber lieben Liebe.

(Weigmann/Bieler/Schenk 2000d: 17)

In den Arbeitsbüchern von Deutsch international finden wir auch explizite grammatische Aufgaben, deren Sprache Kroatisch ist, z. B.: "Spreži glagole." [= Konjugiere die Verben.] (Weigmann/Bieler/Schenk 2000a, AH: 53). In der Aufgabenstellung werden Deutsch und Kroatisch kombiniert:

Koji glagoli tvore perfekt s pomoćnim glagolom haben, a koji sa sein? Dovrši pravilo. ${ }^{12}$

Die meisten Verben bilden das Perfekt mit dem Hilfsverb

Verben, die Fortbewegung oder Zustandsveränderung ausdrücken, und die Verben sein, bleiben und passieren bilden das Perfekt mit (Weigmann/Bieler/Schenk 2000c, AH: 5)

Das Lehrwerk zweite.sprache@deutsch.de (Horvatić Čajko/Lasić 2007a, 2007b, 2008) ist für den Deutschunterricht an Gymnasien und verwandten Schulen bestimmt. Im Lehrbuch für das erste Lernjahr finden wir zuerst Angaben zu den Relationen Laut - Graphem und zur Qualität der Vokale. Angaben zur Grammatik findet man in den Merkzetteln, die schon im ersten Jahr auch die Ebene der Pragmatik berücksichtigen, wie z.B. Ausdrücke der Höflichkeit und Grußformeln. Die Darstellungsgrundlage ist kontrastiv - auf der phonetischen bzw. graphemischen Ebene findet man Beispiele, die sich vom Kroatischen unterscheiden. Weitere Merkzettel sind vor allem der Morphologie gewidmet. Im Merkzettel 3 findet man Präsensparadigmen der Verba mögen, können und möchten (sic!) (vgl. Horvatić Čajko/Lasić 2007a: 25).

Der Ansatz ist teilweise auch kognitiv:

Wie lautet die Regel?

Ratschläge stehen oft im I ... + ... oder man formuliert sie auch mit den Modalverben: ... und ... .

(Horvatić Čajko/Lasić 2007a: 41)

und die Regeln sollen die Lerner auf Deutsch ergänzen, obwohl es sich um das erste Lernjahr handelt:

Die Konjunktion im Kausalsatz ist ... oder .... ... steht am Anfang des Kausalsatzes, wenn der Kausalsatz an 1. Stelle steht. (Horvatić Čajko/Lasić 2007a: 42)

Es überwiegt aber der traditionelle präskriptive Ansatz.

Im zweiten Jahr wird dann auch die pragmatische Ebene eingeführt, es werden Ausdrucksmittel für Ratschläge, Vorschläge und Höflichkeit vermittelt. Man unterscheidet bei den Modalverben Indikativ Präteritum und Konjunktiv Präteritum als Höflichkeitsform, woraus ersichtlich ist, dass ich möchte usw. das Konjunktivparadigma des Präteritums von mögen ist, also dass die Angaben im Merkzettel 3 für das erste Lernjahr nicht stimmen. Im Merkzettel 4 werden dann die Konjunktiv Präteritum Paradigmen von dürfen, können, mögen, müssen, sollen und wollen angeführt (vgl. Horvatić Čajko/Lasić 2007a: 61); möchten ist verschwunden. Im Lehrbuch für das dritte Lernjahr wird auf die Rektion der Verba hingewiesen.

Zum Gebrauch des Plusquamperfekts werden Beispiele ohne Kontext angeführt:

Plusquamperfekt in einem einfachen Satz: Ich hatte einen alten Freund getroffen. (Horvatić Čajko/Lasić 2008: 19)

\subsection{Fazit}

Die meisten der analysierten Lehrwerke beruhen auf den Prinzipien der traditionellen Grammatik - von den drei sprachlichen Ebenen werden allgemein vor allem die grammatischen

12 Übersetzung: Welche Verben bilden das Perfekt mit dem Hilfsverb haben und welche mit sein? Vervollständige die Regel. 
Einheiten der morphologischen und der syntaktischen Ebene berücksichtigt. In den Lehrwerken für die Grundschule findet man dagegen mehr Hinweise und Übungen zur phonetischen und graphemischen Ebene.

Die von den kroatischen Autoren verfassten Lehrwerke haben einen betont kontrastiven Ansatz. In allen Lehrwerken wird auch die pragmatische Ebene berücksichtigt, obwohl meistens nur ansatzweise. Die Textebene spielt nur in Kontaktsprache Deutsch nue (Häusler 2004; Häusler/Bračun/Kovačić 2001; Häusler/Kern-Francetić 1998, 2000) eine Rolle. Obwohl die meisten Lehrwerke auf die Rektion des Verbs hinweisen, werden die Vorteile der Dependenzgrammatik im kontrastiven Vergleich nicht berücksichtigt - gerade die Dependenzgrammatik wäre geeignet, die Grundzüge der Bildung unterschiedlicher Satzmuster zu erklären, sowie auch die Relationen zwischen den Satzgliedern, die wegen der Interferenz häufig gleiche Fehler verursachen.

Die meisten AutorInnen haben Schwierigkeiten, wenn sie in ihren Lehrwerken Grammatikbücher imitieren wollen. Viele Definitionen sind unpraktisch oder schwer verständlich. Diese Vorliebe für Grammatiknachahmung zeigt sich auch in den gesonderten Grammatiktafeln, die als Anhang zu einzelnen Lektionen oder als Anhang zum gesamten Lehrwerk vorkommen.

Hervorzuheben ist auch der kognitive Ansatz, der entweder auf induktiven oder deduktiven grammatischen Erläuterungen bzw. Aufgaben beruht, die je nach der Kompetenz der Lerner auf Kroatisch oder Deutsch bzw. mit Übersetzung realisiert werden.

\section{$4 \quad$ Fachdidaktische Analyse}

Für die fachdidaktische Analyse der Grammatikvermittlung in neueren kroatischen DaFLehrwerken wurden folgende Aspekte untersucht: Lernziele und die Progression grammatischer Inhalte, funktionale Aspekte und die Kontextualität, Erarbeitung und Bewusstmachung grammatischer Phänomene, die Regeldarstellung und die Übungen.

\subsection{Lehrwerke und Zielkompetenzen}

Die fachdidaktische Analyse wurde an Lehrwerken für Anfänger in Gymnasien und den übrigen Mittelschulen durchgeführt. Für Lerner, die im Alter von 14 oder 15 Jahren mit Deutsch als zweiter Fremdsprache anfangen, sind drei Lehrwerke zugelassen: Deutsch für heute und morgen (Marčetić 1997-2000, vierstufig, für Klassen 1 bis 4, mit Arbeitsheften und Kassetten); Deutsch international (Weigmann/Bieler/Schenk ab 2000, vierstufig, für Klassen 1 bis 4, mit Arbeitsheften und Kassetten) und zweite.sprache@deutsch.de (Horvatić Čajko/Lasić ab 2007, vierstufig, für Klassen 1 bis 4, mit Arbeitsheften und CDs, Folien und Arbeitsblättern).

Allen drei Lehrwerken ist ein durchdachtes, didaktisch-methodisches Konzept insoweit gemeinsam, als sie nicht nur Unterrichtsmaterialien (Texte, Grammatikdarstellung, Übungen) enthalten, sondern bis ins Detail ausgearbeitete Unterrichtsszenarien entwerfen. Die restlose methodische Aufarbeitung von Unterrichtsmaterialien ist nicht nur von Vorteil: Lustlose Lehrkräfte können einfach dem Lehrwerk folgen, einsatzfreudigen Lehrenden lässt es jedoch kaum Raum für Eigeninitiative, besonders wenn man die Überfrachtung mit grammatischem Lehrstoff in Betracht zieht.

Die Lehrwerke Deutsch für heute und morgen und zweite.sprache@deutsch.de wurden von kroatischen Autorinnen verfasst, dies bedeutet: Ihre Konzeption ist wesentlich geprägt durch die Eigenschaften der Zielgruppen - Muttersprache und die Erfahrungswelt der Lerner - sowie durch institutionelle Gegebenheiten in kroatischen Schulen.

Das Lehrwerk Deutsch international ist die Adaptation eines deutschen Lehrwerks angesehener Autoren. Für den Gebrauch in Kroatien wurden in den Wortlisten am Ende der 
Kursbücher kroatische Entsprechungen hinzugefügt und die Arbeitsanweisungen (Überschriften der Übungen) und metasprachliche Erklärungen ins Kroatische übersetzt.

Ein weiterer Unterschied liegt in den Anforderungen an die Zielgruppe und den angestrebten Zielkompetenzen. Die Lehrwerke Deutsch für heute und morgen und Deutsch international wurden für die Mehrheit der Schüler konzipiert, die mit Deutsch als zweiter Fremdsprache in den Mittelschulen anfangen, lediglich zwei Wochenstunden und insgesamt maximal 280 Stunden Unterricht haben. Diese Lehrwerke wurden verfasst, bevor die Beschreibung der Zielkompetenzen mithilfe von Deskriptoren für die 6 Kompetenzniveaus des Gemeinsamen europäischen Referenzrahmens für Sprachen (engl. und franz. Version 2000, deutsch 2001, kroatisch 2005) allgemeine Anwendung fand. Nach der Zahl der lehrplanmäßig vorgesehenen Unterrichtsstunden würde das angestrebte Kompetenzniveau dieser Lehrwerke den Stufen A2 bis A2+ entsprechen.

Eine Voraussetzung für den Einsatz des Lehrwerks Deutsch für heute und morgen ist eine intensive häusliche Arbeit der Lerner, wozu ihnen das Lehrwerk bestmögliche Hilfe bietet und sie dadurch in die Lage versetzt, auch später ihre Deutschkenntnisse aufzufrischen und zu erweitern, also in gewissem Sinne, durch Arbeitsanweisungen für das Vorgehen beim Erlernen von Fremdsprachen und die Vermittlung allgemeingrammatischer Kategorien, autonomes Lernen fördert.

Das Lehrwerk zweite.sprache@deutsch.de dürfte das Niveau B1 anstreben. Es wendet sich auch an Sprachgymnasien und sonstige Mittelschulen mit mehr Stunden für den Sprachunterricht. Neben einer größeren Stundenzahl (bis etwa 455) sind die Lerner in den Sprachgymnasien geübt im Umgang mit sprachanalytischen Verfahren, sie haben ein größeres Interesse am Sprachenlernen und auch mehr einschlägige Erfahrung. Metasprachliche Kenntnisse, sprachanalytische Fähigkeiten und sprachvergleichende Verfahren (z. B. in Übersetzungen) gehören für viele von diesen Lernern zu Zielkompetenzen, die sie im Studium und später im Beruf brauchen werden.

\subsection{Auswahl und Reihenfolge grammatischer Inhalte}

Für die Auswahl und Anordnung grammatischer Inhalte nach Lernstufen sollten die Lehrwerke dem vom Ministerium vorgeschriebenen Lehrplan folgen (Nastavni program za gimnazije 1994).

Für Deutsch als zweite Fremdsprache sieht der vorgeschriebene Lehrplan in den vier Klassenstufen der Mittelschulen die Vermittlung der ganzen Grundstufengrammatik vor, wobei in den beiden ersten Jahren fast die ganze Formenlehre ansteht. Für die beiden letzten Klassen bleibt von der Formenlehre nur wenig "Lernstoff" übrig: in der 3. Klasse Präpositionen mit dem Genitiv, als Substantiv gebrauchte Adjektive und Partizipien, Pronominaladverbien, Rektion der Verben und das Passiv (alle Zeitformen!!!). Für die 4. Klasse sind aus der Morphologie nur Attribute mit Partizipien und Modalverben im Perfekt und Plusquamperfekt (Konjunktiv 2) vorgesehen. Der Schwerpunkt dieser beiden letzten Klassen vor dem Abitur liegt bei der Syntax. In der 3. Klasse: Temporalsätze mit den Konjunktionen als, wenn, bevor, solange; Finalsätze mit damit und $u m$; Infinitiv mit $z u$; indirekte Fragesätze; Relativsätze. In der 4. Klasse: Irreale Konditionalsätze für Gegenwart und Vergangenheit; indirekte Rede; Komparativsätze mit dem Konjunktiv; Temporalsätze mit den Konjunktionen solange, seitdem, sobald, nachdem, ehe, bis, bevor; Modalsätze und deren Verkürzung (sic!), Konsekutivsätze und deren Verkürzung (sic!).

Diese Aufteilung des grammatischen Lernstoffs mit dem Schwerpunkt der zwei ersten Lernjahre bei der Formenbildung und der Behandlung der Syntax im 3. und 4. Lernjahr finden wir in den kroatischen Lehrwerken für Gymnasien seit etwa 1850, sie ist auch häufig in anderen 
Lehrwerken anzutreffen, geerbt wurde sie von der Aufteilung des grammatischen Lernstoffes für den Lateinunterricht.

Diese Aufteilung, die der systematischen Sprachbeschreibung folgt, ist in didaktischer Hinsicht insoweit problematisch, als dadurch praktisch fast alles, was es für die deutsche Sprache an Grammatik zu "lernen" gibt, in den zwei ersten Lernjahren angehäuft wird, so dass für das dritte und vierte Lernjahr kaum noch etwas übrig bleibt. Für kroatischsprachige Schüler enthält die "Syntax" als Niveau der Sprachbeschreibung nur wenig "Lernstoff". Die wichtigsten Unterschiede zwischen der deutschen und der kroatischen Sprache betreffen die feste Stellung bestimmter Satzteile und die Konstruktionen Infinitiv mit $z u$. Der Gebrauch von Tempora und von Infinitivkonstruktionen wird im Rahmen der Morphologie vermittelt. Wenn beim Modusgebrauch, etwa in der indirekten Rede, zwischen Mitteilungs- und Verstehensgrammatik unterschieden wird, bleibt von der Syntax nur die Wortstellung als eine der allgemein bekannten "Schwierigkeiten der deutschen Sprache" übrig, die jedoch von Anfang an geübt wird. Im Deutschunterricht an kroatischsprachige Lerner ist die Vermittlung von Nebensatzarten als grammatischer Kategorie eine unnötige Erschwernis, da die Lerner die entsprechenden Konzepte von ihrer Muttersprache her beherrschen. Subordinierende Konjunktionen können als lexikalische Einheiten mit jeweiliger Bedeutung vermittelt werden, ohne auf die für das allgemeine Publikum nur schwer verständliche Benennung der Nebensatzarten einzugehen.

Nach der traditionellen Sprachbeschreibung gehören zur Syntax der Gebrauch der Kasus bzw. die Rektion der Verben, einiger Adjektive und Substantive, wodurch auch Pronominal- und Frageadverbien zum Lernstoff der Syntax werden. Sinnvollerweise sollten diese Erscheinungen parallel mit der Behandlung der Formenbildung und der Einführung lexikalischer Einheiten vermittelt werden und nicht als Lernstoff der Syntax. Die Vermittlung wäre wesentlich einfacher, wenn man als Grundlage statt der traditionellen Subjekt-Objekt-Grammatik die Dependenzgrammatik heranziehen würde. Dies würde ein weiteres Problem beseitigen: "Reflexive Verben" brauchten dann nicht als grammatische Kategorie vermittelt zu werden (zumal in Paradigmen lediglich reflexiv gebrauchte Verben konjugiert werden), die Darstellung der Tempusbildung wäre dann einfacher, die Kategorie "Verben mit dem Reflexivpronomen" brauchte man nur bei den Regeln für den Gebrauch von haben und sein als Hilfsverben. Aufgrund der Dependenzrelationen könnte man kontrastiv auch die Satzmodelle erläutern.

Eine steile und lineare Progression der Vermittlung grammatischer Inhalte ist für Deutsch als zweite Fremdsprache in Mittelschulen also schon vom Lehrplan her vorgegeben. Natürlich bliebe bei der Umsetzung viel Spielraum für eine vertretbare Reihenfolge übrig, zumal der Lehrplan nicht zwischen Mitteilungs- und Verstehensgrammatik unterscheidet.

Das Lehrwerk für Anfänger in den Mittelschulen Deutsch für heute und morgen hat eine steile und lineare Progression. So werden z. B. die Pluralendungen der Substantive (einschließlich $-s$ ) schon in der 3. Lektion der ersten Klasse systematisiert, auch der Imperativ wird (wie vom Lehrplan vorgegeben) in der ersten Klasse fast vollständig eingeführt, und zwar in der vorletzten Lektion. Neben sprachsystematischer Motivierung ist die Reihefolge der Einführung grammatischer Strukturen in diesem Lehrwerk stark motiviert durch Themen und Inhalte. Wir erläutern dies am Beispiel der Einführung des Perfekts.

Für die Vergangenheit ist es heute üblich, aus pragmatischen Gründen das Perfekt vor dem Präteritum einzuführen. ${ }^{13}$ Dem Lehrplan gemäß soll in der ersten Klasse das Perfekt "einiger häufig gebrauchter Verben" (Nastavni program za gimnazije 1994: 45) vermittelt werden, also ohne Rücksicht auf deren morphologische Eigenschaften. In der zweiten Klasse ist das Perfekt regelmäßiger und unregelmäßiger Verben und der Verba auf -ieren vorgesehen, für

${ }^{13} \mathrm{Zu}$ den Kriterien für die Reihenfolge der Einführung grammatischer Strukturen vgl. Funk/Koenig1991: 62f. 
die 3. Klasse wird die "Wiederholung, Festigung und Erweiterung" (ebd.: 46) wohl auch der Perfektbildung verlangt.

Im Lehrwerk Deutsch für heute und morgen wird das Perfekt nicht in der ersten, sondern erst in der zweiten Klasse eingeführt. Zunächst, in der 1. Lektion, im Themenzusammenhang "Lese- und Fernsehgewohnheiten heutiger Jugendlicher", wird nur das Verb lesen eingeführt, und zwar als Verstehensgrammatik: Es erscheint im Ausgangstext (Ergebnisse einer Umfrage) und in den personenbezogenen Fragen nach der Textarbeit und Grammatikerarbeitung, wo es im Nebensatz steht: "Frag deine Schulfreunde, was sie in der letzten Zeit gelesen haben" (Marčetić 1998: 11); "Nenne einige Bücher, die du in den letzten Monaten gelesen hast" (ebd.: 18). Das Paradigma des Verbs lesen im Perfekt wird nicht gegeben, stattdessen werden die Lerner angeleitet, in einer halbproduktiven Formationsübung die Formen in Analogie zu den Stimuli zu bilden, auch die Anforderung "Frag deine Freunde ..." verlangt Analogiebildung. Die Einführung des Perfekts beginnt also mit der Erschließung der Frageform des Perfekts von lesen durch die Lerner, während der Gebrauch im Nebensatz, mit Endstellung des Hilfsverbs, als Verstehensgrammatik vermittelt wird.

Nach und nach erscheinen in den folgenden Lektionen, bedingt durch die Auswahl des Themas und der Texte, einige weitere Verben im Perfekt, so z. B. im thematischen Zusammenhang der 2. Lektion "Wohnen und Haushaltspflichten" die Ausdrücke er hat es eingerichtet, er hat wieder geheiratet, sie hat es mit vielen Postern und Pflanzen verschönert. In den Kommentaren werden die Lerner auf diese Formen aufmerksam gemacht. Erst in der 5. Lektion, im Zusammenhang des Themas "Eltern-Kinder-Beziehungen", wird dann die Perfektbildung und die Stellung des Hilfsverbs ausführlich erläutert. Die Autorin erwähnt auch das Verb bringen als zu einer besonderen Gruppe gehörend. In der darauf folgenden Lektion 6 wird näher auf den Gebrauch des Hilfsverbs sein eingegangen, auch ein Verb mit Perfektbildung mit haben oder sein (fahren) wird erwähnt. Man bemerkt die Absicht der Autorin, auch in einem bescheidenen Umfang doch einige Einzelheiten des Formengebrauchs zu berücksichtigen. Bedingt durch die Thema- und Textwahl ist auch die Einführung des Konjunktivs Präteritum von Hilfsverben und einigen Modalverben (Themenzusammenhang: "Partnerbeziehungen und Familie heute"), noch bevor das Präteritum der Präteritopräsentia (Themenzusammenhang "Jugendliche in Ost und West nach der Wiedervereinigung") vermittelt wurde.

Das bestimmende Element der Progression in diesem Lehrwerk ist neben Sprachsystematik ein schülerbezogenes Konzept der Landeskunde: Die Themen und Texte des Lehrwerks enthalten vom Anfang an sorgfältig ausgewählte, landeskundlich relevante Inhalte, so dass die deutsche Sprache anhand kulturspezifischer Inhalte und ohne explizite Vermittlung der Landeskunde gelernt werden kann. Die Inhalte bieten den Lernern gleichzeitig Vergleichsmöglichkeiten und Äußerungsanlässe.

Die Reihenfolge der Einführung grammatischer Erscheinungen in der kroatischen Version des Lehrwerks Deutsch International des deutschen Verlags Cornelsen folgt dem kroatischen Lehrplan nur in manchen Elementen. Hier ist die Progression flach und konzentrisch, d. h. eine grammatische Erscheinung wird nicht auf einmal in ihrer vollen Differenziertheit eingeführt, sondern in zeitlichen Abständen wieder aufgenommen und durch weitere Formen ergänzt. So wird z. B. die Einführung von Kasusformen des bestimmten und unbestimmten Artikels und der Possessivpronomina über das ganze erste Lernjahr verteilt, der Dativ und der Akkusativ der Personalpronomina und das Reflexivpronomen werden aber erst im zweiten Jahr thematisiert.

Doch auch dieses Lehrwerk verschiebt die Einführung des Perfekts weit in das zweite Lernjahr. Die Einführung verschiedener Verbgruppen wird in folgender Weise auf drei Lektionen verteilt: In der ersten Lektion der Perfektvermittlung werden nur regelmäßige Verben einge- 
führt, die das Perfekt mit dem Hilfsverb haben bilden, darunter auch einige Verben mit untrennbaren Präfixen er- und ver-. Die Regelerarbeitung beginnt mit einer einprägsamen graphischen Darstellung der Formenbildung im Satzkontext, wobei die Stellung des Hilfsverbs und des Partizips im Aussagesatz und in Entscheidungsfragen herausgestellt wird. In der darauf folgenden Lektion werden zunächst die Verben mit trennbaren Präfixen thematisiert, anschließend die Verben auf -ieren und einige unregelmäßige Verben. In der letzten Lektion mit Perfekteinführung werden weitere unregelmäßige Verben hinzugefügt und die Perfektbildung mit sein behandelt. Graphisch einprägsam wird die Stellung des Reflexivpronomens im Perfekt reflexiv gebrauchter Verben dargestellt. Diese Aufteilung der Perfekteinführung entspricht mehr der traditionellen als die Anordnung in den kroatischen Lehrwerken. Nicht traditionell ist die unauffällige Einführung von kennen gelernt aus der situativen Einbettung einer produktiven kommunikativen Übung, die an das Thema des Präsentationstextes für das Perfekt ("Feste in Deutschland") anschließt: die Lerner verstehen die Form von der kroatischen Übungsanweisung her: "Upoznao si novu osobu na nekom tulumu. Ispuni tablicu, a zatim opiši osobu u svojoj bilježnici. ${ }^{14}[$...] Ich habe ... bei einer Party kennen gelernt. Er/Sie ist ... ... alt und ... ." (Weigmann/Bieler/Schenk 2000b, AH: 27). Man würde sich wünschen, in den Lehrwerken häufiger grammatische Strukturen anzutreffen, die nicht immer alle thematisiert werden.

Das neueste kroatische Lehrwerk für Anfänger in den Mittelschulen, zweite.sprache@deutsch.de, hat eine sehr steile und lineare Progression. Schon in der zweiten Hälfte des ersten Buches werden auch stärker differenzierte grammatische Phänomene in vollem Umfang nach systemsprachlicher Art vermittelt, so etwa der Imperativ. Noch deutlicher wird die Linearität bei der Einführung des Perfekts. Auch dieses Lehrwerk lässt die Lerner ein ganzes Jahr Deutsch lernen, ohne ihnen die Gelegenheit zu bieten, sich über etwas Vergangenes oder Abgeschlossenes zu äußern. In der ersten Lektion des zweiten Buches wird dann fast die vollständige Perfektbildung, soweit sie zur Grundstufengrammatik gehört, vermittelt: regelmäßige und unregelmäßige Verben, Verben mit betonten und mit unbetonten Präfixen, Verba auf -ieren, sowie der Gebrauch von haben oder sein. In der graphischen Regeldarstellung wird jedoch die Satzklammer nicht betont, es finden sich auch keine Beispielsätze für Perfektformen in Fragesätzen und im Nebensatz. Das Lehrwerk konzentriert sich also ausschließlich auf die Bildung der Formen. In der an die Regeldarstellung angeschlossenen Übung werden als Stimuli 28 (!) Ausdrücke mit dem Infinitiv vorgegeben, die in einem Bericht über den gestrigen Tag im Perfekt gebraucht werden sollen, z. B. "8 Stunden schlafen (h. geschlafen); um 6.30 Uhr aufstehen (b. aufgestanden)" (Horvatić Čajko/Lasić 2007b: 11).

Da Deutsch als flexionsreiche Sprache gilt, liegt der Schwerpunkt der Grammatikvermittlung in den Lehrwerken für die Grundstufe in der Formenlehre. In den Lehrwerken kroatischer Autorinnen (Deutsch für heute und morgen und zweite.sprache@deutsch.de) kann man bei der Gewichtung einzuführender Grammatikregeln einige Besonderheiten feststellen: Diese Autorinnen widmen den grammatischen Erscheinungen, die erfahrungsgemäß den kroatischsprachigen Deutschlernern Schwierigkeiten bereiten, verstärkte Beachtung. Dazu gehören vor allem Erscheinungen, die sich von der kroatischen Muttersprache der Lerner abheben, bspw. der Gebrauch des Artikels (bestimmter, unbestimmter oder der Nullartikel), die feste Stellung verbaler Satzteile und des Subjekts, der Gebrauch von Modalverben (deren Bedeutung), der Gebrauch einiger Präpositionen. Diese Erscheinungen werden möglichst früh eingeführt und von Anfang an massiv geübt, wodurch die Autorinnen eine feste kognitive Verankerung solcher Strukturen als Unterstützung deren Überführung in automatisierte Handlungen anstreben.

\footnotetext{
14 Übersetzung: Auf einer Party hast du eine Person kennen gelernt. Fülle in der Tabelle die Angaben zu dieser
} Person aus und beschreibe sie dann. 


\subsection{Funktionale Aspekte der Grammatik, Kontextualität}

Moderne Lehrwerke vermitteln grammatische Strukturen unter dem Aspekt ihrer Leistung für die Kommunikation. Grammatische Phänomene werden in Texten und thematischen Zusammenhängen präsentiert, geübt und gefestigt, und zwar so "wie es im Hinblick auf ihre Anwendung in der Sprachpraxis sinnvoll erscheint" (Heyd 1990: 163).

Im Lehrwerk Deutsch für heute und morgen werden der Gebrauch und die Funktion grammatischer Strukturen in textuellen Zusammenhängen und in situativer Einbettung der Übungen veranschaulicht und in verbal formulierten Erläuterungen verdeutlicht. Die Funktion wird auch bei der Textwahl berücksichtigt. So enthält zum Beispiel die Lektion, in welcher die Bildung des Imperativs im thematischen Zusammenhang "Nahrungsmittel, Vorbereitungen für eine Party" vermittelt wird, als Lesetext ein Rezept mit Infinitiven, wodurch die Lerner mit einer weiteren Möglichkeit Aufforderungen zu formulieren bekannt gemacht werden.

Funktionale Aspekte der Grammatik treten im Lehrwerk deutscher Autoren, Deutsch international, stärker in den Vordergrund. So enthalten etwa die kleinen Texte über "Feste in Deutschland", die als Präsentationstexte für die Einführung des Perfekts dienen, auch Beispiele für das Resultatsperfekt, z. B. im Text über den Karneval: "Sie tragen Kostüme und Masken. Manche haben lange für dieses Fest gespart" (Weigmann/Bieler/Schenk 2000b: 30). Auch in den Beispielsätzen für die Erarbeitung der Formen und der Wortstellung wird der Bezug zur Gegenwart deutlich herausgestellt: "Habt ihr Sekt gekauft? Dann können wir Silvester feiern" (Weigmann/Bieler/Schenk 2000b: 31).

Das neueste Lehrwerk für Anfänger in den Mittelschulen, zweite.sprache@deutsch.de, berücksichtigt ebenfalls die Funktion grammatischer Strukturen. So wird z. B. der Imperativ zusammen mit den Präsensformen von dürfen und müssen im thematischen Zusammenhang "Kranksein und geund leben" eingeführt. Die gemeinsame Aufforderungsbedeutung wird auch der Regelerarbeitung und den Übungen zugrunde gelegt ("Ivan gibt Anja Ratschläge", Horvatić Čajko/Lasić 2007a: 41).

Der funktionale Aspekt der Grammatik wird in diesem Lehrwerk manchmal vernachlässigt. So wird in einem Dialog über Sommerferien (Horvatić Čajko/Lasić 2007b: 8) das Perfekt nur in seiner Bedeutung des vergangenen Geschehens präsentiert. Da die Autorinnen lediglich die Bildung der Perfektformen beachten, kommt es in den Übungen zu unspezifischer Verwendung der Perfektformen. So sollen die Lerner in zusammenhängenden Berichten über die Sommerferien und über den gestrigen Tag die Lücken mit den Perfektformen ausfüllen. In einer weiteren Übung sollen sie einen Bericht über Alltagsgewohnheiten einer Person ins Perfekt setzen. Dadurch wird der Text zu einem Bericht über einen bestimmten Tag. Auf diese Bedeutungsverschiebung werden die Lerner in der Übungsanweisung jedoch nicht aufmerksam gemacht. Die ausschließliche Berücksichtigung der Formenbildung führt auch zu der funktionell undifferenzierten und somit inkorrekten Kannbeschreibung in der Selbstevaluation am Ende der Lektion: "Ich kann über die Vergangenheit (im Perfekt) erzählen. Ich kann über meinen Alltag im Perfekt erzählen." (Horvatić Čajko/Lasić 2007b, AH: 15)

\subsection{Erarbeitung und Isolierung grammatischer Strukturen}

Moderne Lehrwerke sehen in der Regel einen induktiven Weg der Einsichtnahme in grammatische Regelmäßigkeiten vor: Grammatische Erscheinungen werden in Texten präsentiert, nach einer oder zwei Übungen zur Textarbeit werden die Lerner angehalten, in Ausgangstexten und weiteren Beispielen nach Analogien zu suchen und die Regeln quasi selbst zu erschließen.

Das Lehrwerk Deutsch für heute und morgen, konzipiert für eine bescheidene Stundenzahl und im Hinblick auf häufig ungünstige institutionelle Bedingungen des Unterrichts von 
Deutsch als zweiter Fremdsprache, gibt in kroatischer Sprache genaue Anweisungen für die Arbeit mit dem Lehrwerk, und zwar mit extensivem Gebrauch linguistischer Termini.

Das Lehrwerk geht von der Annahme aus, dass es ohne eine intensive häusliche Arbeit unter den gegebenen ungünstigen Umständen den Lernern unmöglich ist, eine vertretbare deutschsprachige Kompetenz zu erreichen. Diese Ansicht kann man als das Bestreben interpretieren, bei den Lernern die Voraussetzungen zum autonomen Lernen zu entwickeln und sie dadurch zu lebenslangem Weiterlernen zu befähigen. Dazu gibt ihnen das Lehrwerk viele Hilfen, angefangen bei der Bewusstmachung der Zielsetzungen. So werden im Arbeitsheft, einleitend zu jeder Lektion, unter der Überschrift "Što ćete naučiti u ovoj cjelini?" [= Was lernen Sie in dieser Lektion?] detailliert die Lernziele formuliert: Die Themen der Texte und deren Relevanz für die Lernziele werden erläutert, gelegentlich werden die Inhalte der Texte zusammengefasst, die Quellen und Textsorten angegeben und die Wahl der Texte begründet, meistens durch darin enthaltene landeskundliche Bezüge. In einer übersichtlichen Darstellung werden der nach Sachgebieten gegliederte Wortschatz (Wörter und Wendungen mit kroatischen Entsprechungen) und grammatische Schwerpunkte angeführt.

Für jeden Text und jede Übung gibt die Autorin genaue Anweisungen für das Vorgehen bei der Erarbeitung, z. B.:

Rad s tekstom

Tekst je podijeljen na dva dijela (Erstes Beispiel/Zweites Beispiel), pa je najbolje obraditi dio po dio. Većina novih riječi u frazama prevedena je u uvodu. Naučite imenice skupa s članom i množinom, a glagole u trećem licu prezenta (vidjeti gramatički pregled). Preostale nepoznate riječi nađite u rječniku.

Erstes Beispiel

U ovoj se cjelini pojavljuju brojni glagoli s naglašenim predmetkom. Njihovo je obilježje odvajanje prefiksa u prezentu od osnove i položaj na kraju rečenice. Stoga treba pomnjivo pročitati cijelu rečenicu kako se ne bi pogrešno shvatio glagol, npr. stehen znači stajati, a aufstehen ustati. [...] Vježbajte uporabu glagola aufstehen i mitnehmen proširujući osnovnu rečenicu drugim rečeničnim dijelovima:

Primjer: Ich stehe auflfrüh/jeden Tag/sehr. (Marčetić 1997, AH: 46) ${ }^{15}$

In diesen Verarbeitungsanweisungen werden neben der Formenbildung auch der Gebrauch und die Funktion der Formen erläutert, wobei häufig Bezug auf die Muttersprache der Lerner genommen wird, z. B.:

Većina imenica u ovome tekstu su tvarne imenice: Brot, Butter, Marmelade, Margarine, Käse itd. Budući da su posrijedi imenice koje ne znače određenu količinu, one se u njemačkome jeziku rabe bez člana $[\ldots]$.

\footnotetext{
15 Übersetzung:

Textarbeit

Der Text besteht aus zwei Teilen (Erstes Beispiel/Zweites Beispiel). Bearbeiten Sie zunächst den ersten und dann den zweiten Teil. Für die meisten neuen Wörter aus den Phrasen finden Sie in der Einleitung die Übersetzung. Lernen Sie die Substantive immer mit dem Artikel und der Pluralendung und die Verben mit der 3. Person Singular Präsens (siehe die Grammatikübersicht). Die übrigen unbekannten Wörter schlagen Sie im Wörterbuch nach.

Erstes Beispiel

In dieser Lektion erscheinen viele Verben mit einem betonten Präfix. Im Präsens wird das Präfix getrennt, es steht am Ende des Satzes. Deshalb müssen Sie den ganzen Satz genau lesen, damit Sie das Verb nicht falsch verstehen, denn z. B. stehen bedeutet stajati und aufstehen ustati. [...]

Üben Sie den Gebrauch von Verben aufstehen und mitnehmen, indem Sie den Kernsatz durch weitere Satzglieder ergänzen:

Beispiel: Ich stehe auf./früh/jeden Tag/sehr.
} 
Tvarne imenice uz imenice koje označuju količinu ne stoje - kao u hrvatskome jeziku - u genitivu ( $\check{c} a \check{a} a$ mlijeka), već u obliku jednakome nominativu, bez člana.

Međutim, ipak treba naučiti članove tih imenica jer se u kontekstu u kojemu se misli na određenu količinu mora uporabiti član, npr. Wo ist das Brot? Tu se pita za kruh koji je kupljen i spremljen. (ebd.: 47f.) $)^{16}$

In diesem Lehrwerk werden isolierte Sprachelemente gelegentlich vor der Textpräsentation erläutert und auch geübt, z. B.:

Prije no što počnete slušati (ili čitati) dijaloge, naučite riječi iz uvoda i prijedloge $\mathrm{s}$ dativom $\mathrm{i}$ akuzativom te imperativ iz gramatičkoga pregleda. (ebd.: 50$)^{17}$

Im Lehrwerk Deutsch international werden nach der Textarbeit neue grammatische Strukturen in Übungen und bewusst machenden Verfahren isoliert. Bei einfacheren Strukturen lösen die Lerner ohne explizite Regelvermittlung eine Übung, in welcher sie die neuen Formen einsetzen, z. B. "Dopunite rečenice ličnim zamjenicama. 1. Dirk hat Geburtstag. Alle Freunde feiern mit $_{(\mathrm{D})}$..." (Weigmann/Bieler/Schenk 2000b: 27). ${ }^{18}$

Erscheinungen, die differenzierter vermittelt werden, werden erst nach der Bewusstmachung geübt. Die Bewusstmachung erfolgt schrittweise bei möglichst starker Eigentätigkeit der Lerner. So werden die Lerner z. B. für das Perfekt durch drei als Übungen gestaltete Schritte geleitet, die Regeln selbst zu erschließen: Im ersten Schritt werden Beispielsätze aus dem Text isoliert und mit herausgestellten Charakteristika (eine zusammengesetzte Tempusform, die Satzklammer) abgedruckt. Im zweiten Schritt sollen die Lerner zu gegebenen Partizipien Infinitive suchen, im dritten die Regel formulieren: "Perfekt s pomoćnim glagolom haben. Zapišite pravilo u bilježnicu. Das Perfekt bildet man mit: ... (konjugiert) + ... II" (Weigmann/Bieler/Schenk 2000b: 31). ${ }^{19}$ Zuletzt sollen die Lerner in einer reproduktiven Übung die gehörten Texte wiedergeben und dabei die aufgelisteten Partizipien gebrauchen. Die so erarbeiteten Regeln werden in der "Zusammenfassung" am Ende der Lektion schematisch dargestellt und auch verbal formuliert.

Im Arbeitsheft werden die Regeln noch einmal verbal formuliert und gegebenenfalls ergänzt, wobei die Lerner die wichtigsten Angaben selbst eintragen müssen, z. B.:

Naputak: Particip perfekta pravilnih glagola je jednostavan: na početku stoji a na kraju (e) t. $[\ldots]$

Mjesto pomoćnog glagola i participa perfekta u izjavnim rečenicama

Dopuni pravilo. Kao i modalni glagoli pomoćni glagol stoji na na (Weigmann/Bieler/Schenk 2000b, AH: 29) ${ }^{20}$ Particip perfekta stoji

\footnotetext{
16 Übersetzung: Die meisten Substantive in diesem Text sind Stoffbezeichnungen: [...]. Stoffbezeichnungen, die eine nicht näher bestimmte Menge bezeichnen, werden ohne Artikel gebraucht [...].

Wenn diese Substantive mit einer Mengenbezeichnung gebraucht werden, stehen sie ohne Artikel, in einer dem Nominativ gleichen Form, und nicht, wie in der kroatischen Sprache, im Genitiv ( ̌̌aša mlijeka).

Doch auch für diese Substantive muss man den Artikel lernen, weil er in einem Kontext, in dem eine bestimmte Menge gemeint ist, gebraucht werden muss, z. B.: Wo ist das Brot? Hier wird nach dem gekauften und aufbewahrten Brot gefragt.

17 Übersetzung: Bevor Sie die Dialoge hören oder lesen, lernen Sie aus der Einleitung die Wörter und aus der grammatischen Übersicht die Präpositionen mit dem Dativ und mit dem Akkusativ und die Bildung des Imperativs.

18 Übersetzung: Ergänzen Sie die Sätze durch Personalpronomina.

19 Übersetzung: Das Perfekt mit dem Hilfsverb haben. Notieren Sie die Regel in Ihre Hefte.

20 Übersetzung: Hinweis: Das Partizip Perfekt regelmäßiger Verben ist einfach zu bilden: am Anfang steht und am Ende -(e)t. [...]

Stellung des Hilfsverbs und des Partizips Perfekt in Aussagesätzen

Ergänze die Regel. Wie Modalverben steht auch das Hilfsverb an

Das Partizip Perfekt steht am
} 
Die formbezogenen Übungsanweisungen und grammatische Darstellungen werden sowohl im Lehrbuch als auch im Arbeitsbuch in der Regel kroatisch formuliert, inhaltsbezogene Anweisungen in der Regel deutsch.

Auch im Lehrwerk zweite.sprache@deutsch.de erfolgt die Bewusstmachung grammatischer Regeln vor der Einübung. Nach dem Ausgangstext, der meistens mehrere neue Strukturen enthält, gibt dieses Lehrwerk häufig weitere kleine Texte zur Präsentation einzelner Strukturen. An der Erarbeitung und Bewusstmachung der Regeln ist die Beteiligung der Lerner vorgesehen, ähnlich wie im Lehrwerk Deutsch international: Die Lerner werden durch mehrere Übungen angeleitet, die Regeln selbst zu erschließen und sie anschließend schematisch darzustellen oder verbal zu formulieren. Die farbig unterlegten und eingerahmten Beispielund Mustersätze, Merksätze, schematische Darstellungen oder verbale Formulierungen werden nur teilweise vorgegeben, sie sollen vom Lerner ergänzt werden, z. B.:

PERFEKT $=$ Hilfsverb $\ldots$ oder $\ldots$ (im Präsens) + Partizip Perfekt.

Das Hilfsverb sein gebraucht man bei der Perfektbildung nur mit Verben der Orts- (z. B. ...)

oder Zustandsveränderung [...].

Ein regelmäßiges Verb endet im Partizip Perfekt auf ... . (Horvatić Čajko/Lasić 2007b: 9f.)

Die bewusste Einsichtnahme in sprachliche Regeln wird im Arbeitsheft vertieft, doch hier erst nach der Einübung. So wird für das Perfekt die Wiederholung der induktiven Regelableitung an die Übungen angeschlossen, z. B.:

Welche Verben aus der Aufgabe II a) bilden ihr Perfekt mit dem Hilfsverb sein?

Bedeuten diese Verben [...] eine Zustands- oder eine Ortsveränderung?

Welche von diesen Verben sind regelmäßig und welche unregelmäßig? (ebd., AH: 7)

Im Unterschied zum Lehrwerk Deutsch international wird in dem Lehrwerk zweite.sprache@deutsch.de für die Regelformulierung Deutsch als Metasprache gebraucht. Die Metasprache im Kursbuch dieses Lehrwerks (Überschriften der Übungen und die Verarbeitungsanweisungen, grammatische Erklärungen) ist immer Deutsch (bis auf die Hinweise zu Unterschieden oder Ähnlichkeiten kroatischer oder englischer Entsprechungen), im Arbeitsheft der ersten Stufe sind die Überschriften der Übungen und Anweisungen sowie die Selbstevaluation am Ende jeder Lektion zweisprachig (deutsch und kroatisch), in den "grammatischen Merkzetteln" ist die Metasprache kroatisch. Im Arbeitsheft der zweiten Stufe wird Kroatisch nur noch als Metasprache der "grammatischen Merkzettel" gebraucht.

\subsection{Regeldarstellung}

Für die Regeldarstellung werden im Lehrwerk Deutsch für heute und morgen hauptsächlich verbale Formulierungen gebraucht. Thematisierte Formen werden typographisch durch Fettdruck und andere Druckfarben herausgestellt. Flexionsparadigmen werden umrahmt und farbig unterlegt, was die Orientierung im sonst ziemlich unübersichtlich gestalteten Arbeitsheft erleichtert.

Im Lehrwerk Deutsch international erlaubt ein größeres Buchformat (DIN A4), die Regeldarstellung klar und übersichtlich, also benutzerfreundlicher $\mathrm{zu}$ gestalten. Schematische Darstellungen werden von in Worten formulierten Regeln begleitet. Die Herausstellung der thematisierten Formen durch verschiedene Druckfarben (nur im Kursbuch) wird sparsam, mit erkennbarer Funktion (z. B. für Flexionselemente Grün, für Arbeitsanweisungen Lila, für den Wortschatz Blau) und daher effektiver eingesetzt. Dieses Lehrwerk setzt Elemente der Signalgrammatik ein: Um die Wortstellung und die Beziehungen im Satz zu verdeutlichen, werden für nominale Satzteile blaue Rechtecke und für verbale Teile ebenfalls blau gedruckte Ellipsen verwendet. Bei trennbar zusammengesetzten Verben werden die Ellipsen halbiert und an entsprechende Stellen im Satz platziert. 
Im Lehrwerk zweite.sprache@deutsch.de werden für thematische Strukturen und die Regeldarstellung verschiedene Druckfarben, Umrahmungen und farbige Unterlegungen verwendet. Da jedoch diese Mittel auch für andere Zwecke eingesetzt werden, z. B. bei der Wortschatzvermittlung oder in den Übungen, dienen sie in diesem Lehrwerk eher einer besseren Übersichtlichkeit auf raumsparend, mit viel Sprachmaterial zweispaltig bedruckten Seiten des Kursbuchs und auf den Merkzetteln des Arbeitsbuches.

\section{6 Übungen}

In modernen Lehrwerken für den Fremdsprachenunterricht werden grammatische Strukturen meist in situativen oder thematischen Zusammenhängen geübt. Dabei werden die vielfältigen Übungstypen und -formen eingesetzt, wie sie sich im Laufe der langen Geschichte der Erlernung von Fremdsprachen, einschließlich der klassischen Sprachen, herausgebildet haben.

Im Lehrwerk Deutsch für heute und morgen werden die Übungen durch ausführliche Kommentare begleitet, in denen dem Lernenden die Ziele und der Sinn der Übung deutlich gemacht werden, z. B.:

Imenice u tekstu treba, dakako, naučiti u jednini i množini. Provjerite ovom vježbom jeste li to savladali: Pl. Hier sind Nüsse. Sg. Hier ist eine Nuss. Dort sind Bananen. AH: 49$)^{21}$

In diesem Lehrwerk findet man häufig situativ eingebettete Strukturmusterübungen zur Automatisierung der Formenbildung, wobei die Situation thematisch mit Lektionstexten zusammenhängt. Solche Übungen, ein Überbleibsel der audiolingualen und der audiovisuellen Methode, können ausschließlich formbezogen sein, z. B. "Kako glasi imperativ? [= Wie lautet der Imperativ?] die Getränke holen: Hol(e) die Getränke! Holt die Getränke! ein Stück Torte mitnehmen" (Marčetić 1997: 55); oder bei der Verarbeitung auch eine semantische Komponente enthalten, z. B.: "Oblikuj ovakva pitanja i odgovore [= Bilde ähnliche Fragen und Antworten]: [...] Käse mögen/essen - Magst du Käse? Iss ein Stück Käse! 1. Tomatensaft mögen/trinken ." (ebd.)

Zur Sicherung erworbener Kenntnisse wird meistens die Übersetzung in die Zielsprache eingesetzt, also ein Verfahren, durch das man gezielt die Verwendung bestimmter Strukturen veranlassen kann. Übersetzt werden meistens Dialoge oder kleine Texte. In Kommentaren zu den Übersetzungen werden die Lerner in kroatischer Sprache auf Besonderheiten des Gebrauchs oder der Formenbildung aufmerksam gemacht, es werden Hilfen für richtige Lösungen gegeben, und es wird auf die Unterschiede zur kroatischen Sprache hingewiesen, z. B.:

Kasno se dižem ...: njemačka rečenica mora imati subjekt;

nikada ne ...: u njemačkome jeziku samo jedan niječni oblik;

Pojedete li ...: isto kao: jedete li. (Marčetić 1997: 57)22

Das letzt genannte Beispiel macht auf den Unterschied zwischen der deutschen und der kroatischen Sprache hinsichtlich des Verbalaspekts aufmerksam.

In diesem Lehrwerk überwiegen also Übungen mit einem hohen Grad der Steuerung, mitunter findet man auch Übungen mit Einzelsätzen, bei denen der Kontext von Satz zu Satz wechselt. Der Transfer vermittelter Formen beschränkt sich auf personenbezogene Fragen.

21 Übersetzung: Die Substantive aus dem Text sollen selbstredend im Singular und im Plural gelernt werden. Prüfen Sie Ihre Kenntnisse mithilfe folgender Übung.

${ }^{22}$ Kasno se dižem [ = Ich stehe spät auf] .... der deutsche Satz muss ein Subjekt enthalten; nikada ne [ = niemals nicht] ...: In der deutschen Sprache gebraucht man nur eine Negation; Pojedete li [ = Essen Sie auf] .... dasselbe wie: jedete li [ = Essen Sie $]$. 
Im Lehrwerk Deutsch international sind Übungen mit nicht zusammenhängenden Sätzen eher die Ausnahme, auch typische Drillübungen für die Automatisierung der Formenbildung gibt es nicht; sie werden durch kognitivierende Verfahren (etwa Ergänzen einer Übersicht durch Endungen oder einer schematischen Regelformulierung) ersetzt.

Die meisten Übungen in diesem Lehrwerk sind erkennbar pragmatisch situiert, so dass die Schüler beim Lösen der Übung sinnvoll sprachlich handeln können. Die pragmatische Situierung wird jedoch nicht immer in der Überschrift oder dem einleitendem Satz angegeben, die Überschriften geben häufig nur das grammatische Lernziel an, auch wenn sich die Übung zur Herausbildung der Sprechfertigkeit eignet. Hierzu zwei Beispiele:

Umetni glagole u imperativu. [= Setz die Verben im Imperativ ein.]

1. Dirk isst viel Kuchen. Mutter: "Iss nicht so viel! Sonst hast du nachher keinen Hunger."

2. Der Salat ist noch nicht fertig. Mutter: "Dirk, bitte den Salat fertig."

Umetni glagole u imperativu. [= Setz die Verben im Imperativ ein.]

korrigieren - lernen - lesen - machen - schreiben - sprechen

Die Lehrer machen uns ganz nervös. Dauernd müssen wir etwas tun. Sie sagen:

Lernt die Vokabeln, den Text! einen Text! auf Deutsch! _ die Fehler! _ keinen Quatsch.

Wir wollen doch unsere Ruhe! (Weigmann/Bieler/Schenk 2000b, AH: 23)

Die Übungen in diesem Lehrwerk sind stärker inhaltsbezogen, sie sind überwiegend in einem Textzusammenhang (als Dialog oder Bericht) konzipiert und bieten nicht selten auch visuelle Stimuli.

Im Lehrwerk zweite.sprache@deutsch.de werden neue grammatische Strukturen manchmal in Verbindung mit der Entwicklung der Hörfertigkeit geübt, wie im folgenden Beispiel die Präpositionen mit dem Dativ. Im ersten Dialog sollen die Präpositionen und der Artikel dem Hörtext entnommen werden, im zweiten Dialog sind diese abgedruckt, die übrigen Satzteile sollen ergänzt werden:

Wie lautet der Dialog?

A: Wie fährst du zur Schule, Anja?

B: Ich fahre ... Bus.

A: Kommst du ... Schule gleich nach Hause?

B: Nein, ich bleibe kurz ... Marianne, aber ich bin gegen vier Uhr wieder da.

A: Schönen Tag! Tschüs!

Wie lautet der Dialog mit neuen Details?

A: ... Christiane?

B: ... mit der Straßenbahn.

A: ... nach dem Unterricht ...?

B: ...... bei der Oma. ... um fünf $\mathrm{Uhr}$... .

A: Bis später! ...! (Horvatić Čajko/Lasić 2007a: 22)

Das Beispiel oben hat keine themenbezogene Überschrift. Die Übungen in diesem Lehrwerk haben häufig Überschriften, die lediglich Verarbeitungsanweisungen geben (z. B. "Bilde Sätze. Setze die Adjektive in die Sätze ein.") oder nur den formalen Aspekt betonen, obwohl die Übungen situativ angelegt sind und thematisch mit den Lektionstexten zusammenhängen, z. B.:

Aus dem Imperativ-Satz bekommt man leicht einen Satz mit dem Modalverb müssen oder dürfen. Was gehört zusammen?

Bleib im Bett! [...] Du musst im Bett bleiben. [...]

Nun bilde aus den Sätzen mit dem Modelverb müssen oder dürfen einen Imperativ-Satz! Was gehört zusammen? (ebd.: 41) 
In den meisten Übungen hängen die Sätze durch einen situativen Rahmen zusammen, der manchmal Elemente authentischer Kommunikation enthält, so etwa die "Kommunikation im Klassenraum", in deren Rahmen der für kroatischsprachige Lerner schwierige Gebrauch des Verbs sollen geübt wird:

Das sagt unser/e Lehrer/in immer wieder:

A. Sprich, bitte, Deutsch! B. Schlag das Buch auf Seite 14 auf! C. Schlagt, bitte, euere Hefte auf! [...]

Sagt er/sie noch etwas? Berichte, was die Lehrerin sagt.

A. Die Lehrerin sagt, ich soll Deutsch sprechen. [...] C. Die Lehrerin sagt, wir sollen ... . D. Die Lehrerin sagt, wir ... . E. Die Lehrerin sagt, ich ... . (ebd.: 74)

Kommunikative Situationen, in deren Rahmen die Sprache im realitätsnahen Gebrauch geübt werden könnte, können im Unterricht nur simuliert werden. Das Unterrichtsgeschehen bietet jedoch die Möglichkeit, den Übungen kommunikativen Sinn zu geben. So bekommt die Übung für dass-Sätze in der "Kommunikation im Klassenraum" einen situativ-pragmatischen Rahmen: Die Schüler machen ein Interview mit der Lehrerin und berichten anschließend: "Sie sagt, dass ... ." (Horvatić Čajko/Lasić 2007a: 75).

Ein besonderes Kennzeichen des Lehrwerks zweite.sprache@deutsch.de ist der Verstoß gegen eine verbreitete und meistens auch akzeptierte Empfehlung, in grammatischen Übungen nur bekanntes lexikalisches Material zu gebrauchen. ${ }^{23}$ In vielen Übungen findet man Wörter, die davor nicht eingeführt wurden, und manche Übungen werden auch dazu genutzt, neues Wortmaterial des gegebenen Lernfelds einzuführen. So werden z. B. für das Lernfeld "Kranksein" (welches mit dem Ausgangstext "Anja ist krank" eröffnet wurde), nach der Präsentation der Körperteile anhand einer Zeichnung, für das zu führende Gespräch mit dem Arzt, also ein Rollenspiel, in welchem die Formulierung der Ratschläge geübt werden soll, 13 mögliche Beschwerdenformulierungen für den Patienten (z. B. "Ich habe Kopfschmerzen, Zahnschmerzen, Schnupfen, Allergie, Ich fühle mich nicht wohl") und 21 Ausdrücke im Infinitiv für die Arztrolle (z. B. "zu viel Süßigkeiten essen, zu laute Musik hören, Nasentropfen nehmen, sich erholen" (ebd.: 43)) angeführt. Bei der Ausarbeitung dieser Übung sind die Schüler nicht nur reagierend tätig, sondern sie müssen sich mit neuem Wortmaterial aktiv auseinandersetzten, um daraus Elemente auszuwählen, die sie in ihren Rollen verwenden wollen, was motivierend wirken dürfte. Solche Übungen kann man als produktiv bezeichnen, da sich die Schüler in die Auswahl der Elemente selbst einbringen können.

In einigen Übungen werden visuelle Stimuli gegeben, so etwa Bildchen zum Tagesablauf einer Lehrwerkperson (vgl. ebd.: 44), mit sprachlicher Darstellung dieses Ablaufs sollen die bis dahin vermittelten Verben im Präsens gefestigt werden. Visuelle Stimuli wirken emotional ansprechend und fördern dadurch die Überführung des kognitiv verankerten Sprachwissens in nicht kognitive Handlungsstrukturen.

Produktive Übungen werden in diesem Lehrwerk meistens als Aufgaben formuliert, in welchen lediglich der Inhalt vorgegeben wird, während die sprachliche Ausgestaltung den Lernern überlassen bleibt, z. B.:

Führe ähnliche Dialoge wie im Text Schlussverkauf.

1. Anja möchte sich ein Top kaufen. Sie braucht Größe 38. Tops gibt es in Weiß, Braun und

Gelb. Anja passt das Top gut. Es kostet 16.99 Euro. Sie kauft sich ein braunes Top. (ebd.: 49)

Erscheinungen, die erfahrungsgemäß den kroatischsprachigen Deutschlernern Schwierigkeiten bereiten (s. oben unter 4.2), werden vom Anfang an verstärkt geübt, entweder isoliert, in kontextlosen Einzelsätzen oder in thematischen Zusammenhängen. Besondere Aufmerksam-

${ }^{23}$ So z. B. Wißner-Kurzawa 1995: 235. 
keit widmen die Autorinnen der Wortstellung. Die Inversion des Subjekts in Aussage- und in Fragesätzen wird häufig geübt, z. B. indem die Lerner aufgefordert werden, Fragen zum Ausgangstext zu bilden (vgl. ebd.: 13), im Konjugationsparadigma für trennbare Verben werden auch Fragesätze angeführt und die Wortstellung xVS nach dem vorangestellten Nebensatz wird verdeutlicht schon bei der ersten Einführung subordinierender Konjunktionen: "Welcher Hauptsatz, a) oder b), passt zum WENN-Satz? 1. Wenn Petar um 6.30 Uhr aufsteht, a) er macht sein Bett. b) macht er sein Bett." (ebd.: 45)

Als letzten Schritt bei der Einübung und Festigung erstmalig vermittelter grammatischer Strukturen und für das gegebene Lernfeld typischer Wendungen setzen die Autorinnen die Übersetzung in die Zielsprache ein, wobei es sich um nicht zusammenhängende, eventuell situativ verbundene Sätze oder um Texte handeln kann. Die zu übersetzenden Sätze enthalten für das Lernfeld relevante Wendungen und Strukturen, die sich von der kroatischen Sprache abheben, z. B.:

1. Vlak za Rijeku polazi u 15 sati. 2. Za pet dana je Božić. 3. U utorak idem s Renatom u kino. 4. U proljeće se radi u vrtu. 5. Rado razgovaram s tobom. 6. U koliko sati počinje film u kinu "Europa"? 7. Na njemačkome se kaže Mai, a na engleskome May. (Horvatić Čajko/Lasić 2007a, AH, S. 53)

[Lösung der Aufgabe:]

1. Der Zug nach Rijeka fährt um 15 Uhr ab. 2. In fünf Tagen ist Weihnachten. 3. Am Dienstag gehe ich mit Renata ins Kino. 4. Im Frühjahr arbeitet man im Garten. 5. Ich unterhalte mich gerne mit dir. 6. Wann/Um wie viel Uhr fängt der Film im Kino "Europa" an? 7. Auf Deutsch sagt man Mai und auf Englisch May. (ebd.: 134)

Von der kroatischen Sprache heben sich in diesem Beispiel folgende Strukturen ab: In den Sätzen 1, 2, 3 und 6 sind die Präpositionen verschieden. In den Sätzen 1 und 5 müssen spezifische Verben gebraucht werden. Durch die Übersetzung subjektloser kroatischer Sätze unter 4 und 7 soll der Gebrauch des Subjektpronomens man geübt werden. Mit Satz 7 können die Schüler lernen, dass der adversativen kroatischen Konjunktion $a$ manchmal die deutsche Konjunktion und entspricht. Wichtig ist auch das Syntagma "auf Deutsch", weil das substantivisch gebrauchte Adjektiv als Sprachbezeichnung im Kroatischen die Kasusendung hat, im Deutschen in dieser Verwendung nicht.

\section{$5 \quad$ Fazit}

Methodisch-didaktisch gehören die untersuchten Lehrwerke der postkommunikativen Ära an, für welche die Weiterentwicklung des kognitiven Ansatzes und des autonomen Lernens charakteristisch ist.

Insgesamt messen die AutorInnen der expliziten Grammatikvermittlung mithilfe grammatischer Kategorien große Bedeutung zu: Die Regelableitung und -darstellung ist in die Lektionsteile integriert, am Ende der Lektionen werden die eingeführten Phänomene zusammenfassend in Übersicht dargestellt und manche Lehrwerke geben im Anhang noch eine systemsprachliche Darstellung der behandelten Grammatik.

In deutlicher Absetzung zur audiovisuellen, aber auch zur kommunikativ-pragmatischen Methode, erfolgt die Bewusstmachung des grammatischen Lernstoffes vor dessen Einübung und Festigung. Drillübungen finden sich nur noch sporadisch, stattdessen werden die Lerner in Übungen angeleitet, die Regeln selbst zu erschließen und ggf. graphisch darzustellen oder verbal zu formulieren.

Zur Weiterentwicklung des kognitiven Ansatzes gehört auch der verstärkte Gebrauch der Muttersprache, zum einen als Sprache der grammatischen Erläuterungen und Übungsanweisungen, zum anderen als Rekursmittel für sprachvergleichende Verfahren bei der 
Grammatikvermittlung und in den Übersetzungsübungen. Die Auswahl und Reihenfolge der Einführung grammatischer Erscheinungen erfolgt auf der Grundlage der traditionellen Grammatik, pragmatische und textlinguistische Elemente spielen keine tragende Rolle bei der Konzeption, sondern werden themenbezogen hinzugefügt. Bedauerlicherweise findet die Dependenzgrammatik nur stellenweise Anwendung.

Die Forderung der 1980er Jahre, die Fähigkeit des autonomen Lernens in die Ziele des Fremdsprachenunterrichts zu integrieren, fand ihren Niederschlag in Lehrwerken der 1990er Jahre in der Hinzufügung besonderer Teile für die Vermittlung von Lernstrategien und die Bewusstmachung des erreichten Lernfortschritts. Einige der nach 2000 verfassten Lehrwerke übernehmen die Verfahren der Selbstevaluation mithilfe von Kannbeschreibungen, wie sie im Anschluss an die Bestimmung von Kompetenzniveaus im Gemeinsamen europäischen Referenzrahmen für Sprachen für die Sprachenportfolios entwickelt wurden.

Abschließend sei die Befürchtung geäußert, dass der im oben dargelegten Sinne verstandene kognitive Ansatz zu einem Übermaß an Grammatikarbeit führen könnte. Bei bescheidener Stundenzahl (Deutsch wird in Kroatien häufig nur mit zwei Wochenstunden unterrichtet), würde sich der Deutschunterricht in Grammatikvermittlung erschöpfen. Wenn noch die Arbeit an der Selbstevaluation der Lerner zusätzliche Unterrichtszeit in Anspruch nimmt, würden die übrigen Aktivitäten, die erst authentisches sprachliches Handeln ermöglichen, entschieden zu kurz kommen.

\section{Literatur}

Diehl, Erika (2000): "Theorien zum Zweitsprachenerwerb: Standortbestimmung des DiGSProjekts". In: Diehl, Erika et al. (eds.): Grammatikunterricht: Alles für der Katz? Tübingen: $25-52$.

Engel, Ulrich et al. (1978): Mannheimer Gutachten zu ausgewählten Lehrwerken Deutsch als Fremdsprache. 2. Aufl. Heidelberg.

Funk, Hermann/Koenig, Michael (1991): Grammatik lehren und lernen. Fernstudieneinheit 1. Berlin.

Heyd, Gertraude (1990): Deutsch lehren. Frankfurt am Main.

Kast, Bernd/Neuner, Gerhard (eds.) (1994): Zur Analyse, Begutachtung und Entwicklung von Lehrwerken für den fremdsprachlichen Deutschunterricht. Berlin usw.

Krumm, Hans-Jürgen (ed.) (1982): Lehrwerkforschung - Lehrwerkkritik Deutsch als Fremdsprache. München.

Nastavni program za gimnazije 1994. Glasnik ministarstva kulture i prosvjete Republike Hrvatske. Posebno izdanje.

Storch, Günther (1999): Deutsch als Fremdsprache - Eine Didaktik. München.

Trim, John/North, Brian/Coste, Daniel (2001): Gemeinsamer europäischer Referenzrahmen für Sprachen: lernen, lehren, beurteilen. Berlin usw.

Wißner-Kurzawa, Elke (1995): "Grammatikübungen". In: Bausch, Karl-Richard/Christ, Herbert/Krumm, Hans-Jürgen (eds.): Handbuch Fremdsprachenunterricht. 3. Aufl. Tübingen/ Basel: 232-235.

Zimmermann, Günther (1990): Grammatik im Fremdsprachenunterricht der Erwachsenenbildung. Ismaning.

\section{Analysierte Lehrwerke}

Bernardi-Britvec, Plamenka/Kruhan, Mira/Salopek, Jadranka (1996): Deutsch? Super! 1. Udžbenik njemačkog jezika za 4. razred osnovne škole [= Lehrwerk der deutschen Sprache für die 4. Klasse der Grundschule]. Mit Arbeitsheft, Lehrerhandbuch und Kassette. Zagreb. 
Bernardi-Britvec, Plamenka/Salopek, Jadranka (1998): Deutsch? Super! 4. Udžbenik njemačkog jezika za 7. razred osnovne škole [= Lehrwerk der deutschen Sprache für die 7. Klasse der Grundschule]. Mit Arbeitsheft, Lehrerhandbuch und Kassette. Zagreb.

Bernardi-Britvec, Plamenka/Salopek, Jadranka (1999): Deutsch? Super! 5. Udžbenik njemačkog jezika za 8. razred osnovne škole [= Lehrwerk der deutschen Sprache für die 8. Klasse der Grundschule]. Mit Arbeitsheft, Lehrerhandbuch und Kassette. Zagreb.

Salopek, Jadranka/Bernardi-Britvec, Plamenka (1998): Deutsch? Super! 3. Udžbenik njemačkog jezika za 6. razred osnovne škole [= Lehrwerk der deutschen Sprache für die 6. Klasse der Grundschule]. Mit Arbeitsheft, Lehrerhandbuch und Kassette. Zagreb.

Salopek, Jadranka/Kruhan, Mira/Bernardi-Britvec, Plamenka (1997): Deutsch? Super! 2. Udžbenik njemačkog jezika za 5. razred osnovne škole [= Lehrwerk der deutschen Sprache für die 5. Klasse der Grundschule]. Mit Arbeitsheft, Lehrerhandbuch und Kassette. Zagreb.

Bernardi-Britvec, Plamenka/Salopek, Jadranka (2007a): Flink mit Deutsch 1. Udžbenik njemačkog jezika za 4. razred osnovne škole (Prva godina učenja) [= Lehrwerk der deutschen Sprache für die 4. Klasse der Grundschule (Erstes Lernjahr)]. Mit Arbeitsheft, CD und Lehrerhandbuch. Zagreb.

Bernardi-Britvec, Plamenka/Salopek, Jadranka (2007b): Flink mit Deutsch 4. Udžbenik njemačkog jezika za 7. razred osnovne škole (Četvrta godina učenja) [= Lehrwerk der deutschen Sprache für die 7. Klasse der Grundschule (Viertes Lernjahr)]. Mit Arbeitsheft, CD und Lehrerhandbuch. Zagreb.

Bernardi-Britvec, Plamenka/Salopek, Jadranka (2007c): Flink mit Deutsch 5. Udžbenik njemačkog jezika za 8. razred osnovne škole (Peta godina učenja) [= Lehrwerk der deutschen Sprache für die 8. Klasse der Grundschule (Fünftes Lernjahr)]. Mit Arbeitsheft, CD und Lehrerhandbuch. Zagreb.

Salopek, Jadranka/Bernardi-Britvec, Plamenka (2007a): Flink mit Deutsch 2. Udžbenik njemačkog jezika za 5. razred osnovne škole (Druga godina učenja) [= Lehrwerk der deutschen Sprache für die 5. Klasse der Grundschule (Zweites Lernjahr)]. Mit Arbeitsheft, CD und Lehrerhandbuch. Zagreb.

Salopek, Jadranka/Bernardi-Britvec, Plamenka (2007b): Flink mit Deutsch 3. Udžbenik njemačkog jezika za 6. razred osnovne škole (Treća godina učenja) [= Lehrwerk der deutschen Sprache für die 6. Klasse der Grundschule (Drittes Lernjahr)]. Mit Arbeitsheft, CD und Lehrerhandbuch. Zagreb.

Vajda, Ivana/Nigl, Karin (2006): Deutsch lernen - Deutsch spielen 4. Udžbenik njemačkoga jezika za 7. razred osnovne škole (4. godina učenja) [= Lehrwerk der deutschen Sprache für die 7. Klasse der Grundschule (4. Lernjahr)]. Mit Arbeitsheft, CD, Lehrerhandbuch und didaktischem Koffer. Zagreb.

Vajda, Ivana/Nigl, Karin (2007): Deutsch lernen - Deutsch spielen 5. Udžbenik njemačkoga jezika za 8. razred osnovne škole (5. godina učenja) [= Lehrwerk der deutschen Sprache für die 8. Klasse der Grundschule (5. Lernjahr)]. Mit Arbeitsheft, CD, Lehrerhandbuch und didaktischem Koffer. Zagreb.

Velički, Damir/Matolek Veselić, Gordana (2007a): Deutsch lernen - Deutsch spielen 1. Udžbenik njemačkoga jezika za 4. razred osnovne škole (1. godina učenja) [= Lehrwerk der deutschen Sprache für die 4. Klasse der Grundschule (1. Lernjahr)]. Mit Arbeitsheft, CD, Lehrerhandbuch und didaktischem Koffer. Zagreb.

Velički, Damir/Matolek Veselić, Gordana (2007b): Deutsch lernen - Deutsch spielen 2. Udžbenik njemačkoga jezika za 5. razred osnovne škole (2. godina učenja) [= Lehrwerk der deutschen Sprache für die 5. Klasse der Grundschule (2. Lernjahr)]. Mit Arbeitsheft, CD, Lehrerhandbuch und didaktischem Koffer. Zagreb.

Velički, Damir/Vitez, Ljubimka (2007): Deutsch lernen - Deutsch spielen 3. Udžbenik njemačkoga jezika za 6. razred osnovne škole (3. godina učenja) [= Lehrwerk der deutschen 
Sprache für die 6. Klasse der Grundschule (3. Lernjahr)]. Mit Arbeitsheft, CD, Lehrerhandbuch und didaktischem Koffer. Zagreb.

Salopek, Jadranka/Tomljenović-Biškupić, Ljerka (2007a): Hurra! Deutsch! 3 Udžbenik njemačkog jezika za 3. razred osnovne škole, treća godina učenja [= Lehrwerk der deutschen Sprache für die 3. Klasse der Grundschule (Drittes Lernjahr)]. Mit Arbeitsheft, CD und Lehrerhandbuch. Zagreb.

Salopek, Jadranka/Tomljenović-Biškupić, Ljerka (2007b): Hurra! Deutsch! 5 Udžbenik njemačkog jezika za 5. razred osnovne škole, peta godina učenja [= Lehrwerk der deutschen Sprache für die 5. Klasse der Grundschule (Fünftes Lernjahr)]. Mit Arbeitsheft, CD und Lehrerhandbuch. Zagreb.

Tomljenović-Biškupić, Ljerka/Salopek, Jadranka (2007a): Hurra! Deutsch! 1 Udžbenik njemačkog jezika za 1. razred osnovne škole, prva godina učenja [= Lehrwerk der deutschen Sprache für die 1. Klasse der Grundschule (Erstes Lernjahr)]. Mit Arbeitsheft, CD und Lehrerhandbuch. Zagreb.

Tomljenović-Biškupić, Ljerka/Salopek, Jadranka (2007b): Hurra! Deutsch! 2 Udžbenik njemačkog jezika za 2. razred osnovne škole, druga godina učenja [= Lehrwerk der deutschen Sprache für die 2. Klasse der Grundschule (Zweites Lernjahr)]. Mit Arbeitsheft, CD und Lehrerhandbuch. Zagreb.

Tomljenović-Biškupić, Ljerka/Salopek, Jadranka (2007c): Hurra! Deutsch! 4 Udžbenik njemačkog jezika za 4. razred osnovne škole, četvrta godina učenja [= Lehrwerk der deutschen Sprache für die 4. Klasse der Grundschule (Viertes Lernjahr)]. Mit Arbeitsheft, CD und Lehrerhandbuch. Zagreb.

Häusler, Maja (2004): Kontaktsprache Deutsch 4 neu. Udžbenik njemačkoga jezika za četvrti razred gimnazije, deveta godina učenja [= Lehrwerk der deutschen Sprache für die vierte Klasse Gymnasiums, neuntes Lernjahr]. Mit Arbeitsheft, CD und Lehrerhandbuch. Zagreb.

Häusler, Maja/Bračun, Milica/Kovačić, Drago (2001): Kontaktsprache Deutsch 3 neu. Udžbenik njemačkoga jezika za treći razred gimnazije, osma godina učenja [= Lehrwerk der deutschen Sprache für die dritte Klasse Gymnasiums, achtes Lernjahr]. Mit Arbeitsheft, Kassette und Lehrerhandbuch. Zagreb.

Häusler, Maja/Kern-Francetić, Divna (1998): Kontaktsprache Deutsch 1 neu. Udžbenik njemačkoga jezika za prvi razred gimnazije, šesta godina učenja [= Lehrwerk der deutschen Sprache für die erste Klasse Gymnasiums, sechstes Lernjahr]. Mit Arbeitsheft, Kassette und Lehrerhandbuch. Zagreb.

Häusler, Maja/Kern-Francetić, Divna (2000): Kontaktsprache Deutsch 2 neu. Udžbenik njemačkoga jezika za drugi razred gimnazije, sedma godina učenja [= Lehrwerk der deutschen Sprache für die zweite Klasse Gymnasiums, siebtes Lernjahr]. Mit Arbeitsheft, Kassette und Lehrerhandbuch. Zagreb.

Marčetić, Tamara (1997): Deutsch für heute und morgen 1. Prva godina učenja. Udžbenik za gimnazije [= Erstes Lernjahr, Lehrwerk der deutschen Sprache für Gymnasien]. Mit Arbeitsheft, Lehrerhandbuch und Kassette. Zagreb.

Marčetić, Tamara (1998): Deutsch für heute und morgen 2. Druga godina učenja. Udžbenik za gimnazije [= Zweites Lernjahr, Lehrwerk der deutschen Sprache für Gymnasien]. Mit Arbeitsheft und Kassette. Zagreb.

Marčetić, Tamara (1999): Deutsch für heute und morgen 3. Treća godina učenja. Udžbenik za gimnazije [= Drittes Lernjahr, Lehrwerk der deutschen Sprache für Gymnasien]. Mit Arbeitsheft und Kassette. Zagreb.

Marčetić, Tamara (2000): Deutsch für heute und morgen 4. Četvrta godina učenja. Udžbenik za gimnazije [= Viertes Lernjahr, Lehrwerk der deutschen Sprache für Gymnasien]. Mit Arbeitsheft und Kassette. Zagreb.

Weigmann, Jürgen/Bieler, Karl-Heinz/Schenk, Sylvie (2000a): Deutsch international 1, Teil A. Udžbenik njemačkoga jezika za gimnazije i srodne škole, I. godina učenja [= Lehrwerk 
der deutschen Sprache für Gymnasien und Mittelschulen, I. Lernjahr]. Mit Arbeitsheft, Kassette und Lehrerhandbuch. Berlin usw.: Cornelsen Verlag. Adaptation für die kroatische Ausgabe von Snježana Rodek. Zagreb.

Weigmann, Jürgen/Bieler, Karl-Heinz/Schenk, Sylvie (2000b): Deutsch international 1, Teil B. Udžbenik njemačkoga jezika za gimnazije i srodne škole, II. godina učenja [= Lehrwerk der deutschen Sprache für Gymnasien und Mittelschulen, II. Lernjahr]. Mit Arbeitsheft und Kassette. Berlin usw.: Cornelsen Verlag. Adaptation für die kroatische Ausgabe von Snježana Rodek. Zagreb.

Weigmann, Jürgen/Bieler, Karl-Heinz/Schenk, Sylvie (2000c): Deutsch international 2, Teil A. Udžbenik njemačkoga jezika za gimnazije $i$ srodne škole, III. godina učenja [= Lehrwerk der deutschen Sprache für Gymnasien und Mittelschulen, III. Lernjahr]. Mit Arbeitsheft und Kassette. Berlin usw.: Cornelsen Verlag. Adaptation für die kroatische Ausgabe von Snježana Rodek. Zagreb.

Weigmann, Jürgen/Bieler, Karl-Heinz/Schenk, Sylvie (2000d): Deutsch international 2, Teil B. Udžbenik njemačkoga jezika za gimnazije i srodne škole, IV. godina učenja [= Lehrwerk der deutschen Sprache für Gymnasien und Mittelschulen, IV. Lernjahr]. Mit Arbeitsheft und Kassette. Berlin usw.: Cornelsen Verlag. Adaptation für die kroatische Ausgabe von Snježana Rodek. Zagreb.

Horvatić Čajko, Irena/Lasić, Irena (2007a): zweite.sprache@deutsch.de 1. Udžbenik njemačkoga jezika za 1. razred gimnazija i strukovnih škola, prva godina učenja [= Lehrwerk der deutschen Sprache für die erste Klasse Gymnasien und Fachschulen, erstes Lernjahr]. Mit Arbeitsheft, Lehrerhandbuch, CD, Folien und Arbeitsblättern. Zagreb.

Horvatić Čajko, Irena/Lasić, Irena (2007b): zweite.sprache@deutsch.de 2. Udžbenik njemačkoga jezika za 2. razred gimnazija i strukovnih škola, druga godina učenja [= Lehrwerk der deutschen Sprache für die zweite Klasse Gymnasien und Fachschulen, zweites Lernjahr]. Mit Arbeitsheft, Lehrerhandbuch, CD, Folien und Arbeitsblättern. Zagreb.

Horvatić Čajko, Irena/Lasić, Irena (2008): zweite.sprache@deutsch.de 3. Udžbenik njemačkoga jezika za 3. razred gimnazija i strukovnih škola, treća godina učenja [= Lehrwerk der deutschen Sprache für die dritte Klasse Gymnasien und Fachschulen, drittes Lernjahr]. Mit Arbeitsheft, Lehrerhandbuch, CD, Folien und Arbeitsblättern. Zagreb. 